\title{
Strong and weak coupling of two coupled qubits
}

\author{
Elena del Valle* \\ School of Physics and Astronomy, University of Southampton, SO17 1BJ, Southampton, United Kingdom
}

(Dated: October 3, 2018)

\begin{abstract}
I investigate the dynamics and power spectrum of two coupled qubits (two-level systems) under incoherent continuous pump and dissipation. New regimes of strong coupling are identified, that are due to additional paths of coherence flow in the system. Dressed states are reconstructed even in the regime of strong decoherence. The results are analytical and offer an exact description of strong-coupling in presence of pumping and decay in a nontrivial (nonlinear) system.
\end{abstract}

\section{INTRODUCTION}

Quantum information processing [1] presupposes a coherent coupling of the fundamental bricks of quantum information, the qubits. In real systems, however, decoherence, dissipation and incoherent coupling to the environment is unavoidable [2]. In the field of cavity quantum electrodynamics [3], the notion of coherent coupling is known as strong coupling, in this case, between light and matter (e.g., the excited state of an atomic transition [4] or an electron-hole pair in a semiconductor [5]). This leads to a quantum superposition of the bare states, resulting in so-called dressed states [6]. This regime is reached in systems of very high quality and under tight experimental control, so that intrinsic sources of decoherence are minimized as much as possible and coherent dynamics takes over. The simplest description of strong coupling neglects dissipation altogether and thus reduces to that of mere coupling with strength $g$, introducing the notion of Rabi splitting [7]. Next step in the description includes the decay $\gamma_{i}$ of the bare states, $i=1,2$ [8]. This gives rise to a widely known criterion for strong-coupling: $4 g>\left|\gamma_{1}-\gamma_{2}\right|$. This is the case of vacuum Rabi splitting where at most one excitation is involved. At this level, which is the most natural and fundamental since it describes one particle, there is no difference from the underlying theoretical model. Differences appear at the next step of description when the excitation scheme is taken into account. A typical description of excitation is to consider an initial condition. The coupling is then studied as the spontaneous emission from this initial excited state. Unless the initial condition is restricted to one excitation (as previously), the underlying theoretical model becomes determinant. Another important description of the excitation process is that of a continuous pumping, for instance a coherent excitation that drives the system [9, 10], or an incoherent pump that feeds excitations at a given rate but without any coherent input [11, 12]. The latter is more directly related to the intrinsic dynamics of the system, and is the one that will be considered in this text. With non-negligible pumping, the underlying theoretical description cannot be ignored, and taking it into account leads to strong deviations from the paradigm of strong-coupling as established by the spontaneous emission of one excitation (in any model) [13.

*Electronic address: elena.delvalle.reboul@gmail.com

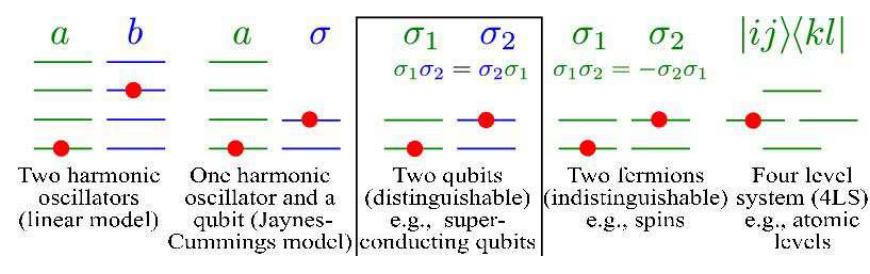

FIG. 1: (Color online) Schema of the systems of interest in this text. The main object of study, two coupled qubits, is sketched in (c). It will be compared throughout with other coupled systems, (a), (b), (d) and (e).

An immediate extension of light-matter coupling in the linear regime is the linear model, namely, that of two harmonic oscillators with no restriction in the number of particles Fig. 11a). Its comprehensive description under incoherent pumping was given in Ref. [14]. This describes for instance exciton-polaritons in planar semiconductor microcavities [5] (where both excitons and photons are bosons). It was shown in this work how pumping calls for extended definitions of strong-coupling, essentially requiring absorption of the pumping rates in the decay rates. A more important theoretical model, known as the Jaynes-Cummings model [15], describes the coupling of a two-level system (such as an atom or a zero-dimensional exciton in a small quantum dot) with a boson mode (typically, cavity photons), Fig. 1 (b). It is more important because more closely related to a genuinely quantum regime, the linear model being essentially a classical description cast in quantum-mechanical terms. Its description under incoherent pumping was given in Ref. [16], but encountered various difficulties to offer a complete picture. In particular, dressed modes exhibit complex patterns and a definition of strong-coupling in this system is much more difficult to achieve, since splitting of the dressed states depends on the excitations. In particular, strong-coupling can be enforced by pumping, leading to situations of mixed weak and strong coupling, where some of the states are bare while some others are dressed.

In this text, I will address the case of two two-level systems (Fig.1 (cde)), which compromises between simplicity of the linear model and richness of the Jaynes-Cummings model. In particular, thanks to the reduced size of the Hilbert space, I will be able to solve the problem fully analytically, as in the linear model, a convenience not afforded by the JaynesCummings model (when including incoherent pumping). This will allow me to provide a complete picture of weak and 
strong coupling in a nontrivial system, and therefore shed light on more complicated systems.

My description will address more particularly independent qubits (Fig. 1 (c)), for instance superconducting (Josephson) qubits [17], in the sense that their commutation rules will not be those of two fermions, that anticommute (Fig. 11d)). I will also address the latter case for comparison and completeness, and obtain the elegant result that the expressions describing two coupled fermions are essentially identical to those describing two coupled bosons, although these two systems are very different in character and behavior (for instance bosons accumulate arbitrary number of particles whereas fermions saturate at at most one, a distinction recovered in the formalism by merely substituting effective parameters). Also because two two-level systems can be mapped to one four-level system (Fig. 1 (e)), I will address this case in detail, finding another fundamental case of interest.

The main results, however, and the deepest connections to be made with other models (such as the Jaynes-Cummings) will be obtained from the case of two qubits. Beyond its interest for the previous reasons, the study of the coupling between two qubits is interesting in its own right [18-21]: it comes as the fundamental support of entangled states [22, 23], to implement quantum gates [24, 25] and, in this quantum information processing context, the natural model to investigate decoherence [26, 27]. In this text, the two coupled qubits will allow us to investigate strong coupling under incoherent pumping.

The rest of this paper is organized as follows. In section II, I introduce the model and its parameters and discuss the level structure. In section III I obtain the single-time dynamics, that will be shown to be the same independently of the underlying model. In section IV I obtain the power spectra, that, in contrast with the previous section, depend strikingly on the underlying model. Power spectra (photoluminescence spectra in a quantum optical context) are important because this is where the dressed states manifest. I will analyze in detail the case of two qubits (Sec. IVA) and contrast it with that of two fermions (Sec. IVB) and of a four-level system (Sec. IV C). In section $\mathrm{V}$ I describe the strong and weak coupling regimes, first in the absence (Sec. $\mathrm{VA}$ ), and then including $\mathrm{VB}$ the incoherent continuous pump. I define new regimes of strong coupling, proper to the two qubits system. In section VI, I illustrate the results of previous sections with examples of some interesting configurations: cases where pumping effect is optimal (Sec. VIA) and detrimental (Sec. VIB) for the coherent coupling. In section VII based on the previous results, I reconstruct the dressed states, uncovering an unexpected manifestation of decoherence-the emergence of additional dressed states - to be found only in the coexistence of pumping and decay along with the coherent coupling. Finally, in section VIII I give a summary of the main results as my conclusions. To avoid distraction in the main text, most technical details appear in appendices $(\mathrm{B}, \mathrm{C}, \mathrm{D})$ along with further material outside the scope of this study $\mathrm{A} \mathrm{E}$.

\section{THEORETICAL MODEL}

The Hamiltonian for two coupled qubits reads:

$$
H_{0}=\omega_{1} \sigma_{1}^{\dagger} \sigma_{1}+\omega_{2} \sigma_{2}^{\dagger} \sigma_{2}+g\left(\sigma_{1}^{\dagger} \sigma_{2}+\sigma_{2}^{\dagger} \sigma_{1}\right)
$$

where $\sigma_{1,2}$ are the lowering operators of the qubits, with bare energies $\omega_{1,2}$. They are linearly coupled with strength $g$. The two modes can be detuned, by a quantity $\Delta=\omega_{1}-\omega_{2}$, that is small enough, $\Delta \ll \omega_{1,2}$, so that the rotating wave approximation is justified. The Hilbert space of the coupled system has dimension four, with the structure $2 \otimes 2$. It can be decomposed in three subspaces (also called manifolds, rungs, etc.) with a fixed number of excitations: the ground state, $\{|0,0\rangle\}$, with zero excitation, the excited state of each qubit, $\{|1,0\rangle,|0,1\rangle\}$, with one excitation, and the state $\{|1,1\rangle\}$ with two excitations.

An important point throughout this text is the commutation rules in Eq. (1), that are those of two distinguishable systems, i.e.,

$$
\begin{aligned}
& \sigma_{i} \sigma_{i}=\sigma_{i}^{\dagger} \sigma_{i}^{\dagger}=0, \quad i=1,2, \\
& {\left[\sigma_{i}, \sigma_{i}^{\dagger}\right]_{+}=\sigma_{i} \sigma_{i}^{\dagger}+\sigma_{i}^{\dagger} \sigma_{i}=1, \quad i=1,2,} \\
& {\left[\sigma_{i}, \sigma_{j}^{\dagger}\right]=\sigma_{i} \sigma_{j}^{\dagger}-\sigma_{j}^{\dagger} \sigma_{i}=0, \quad i \neq j,} \\
& {\left[\sigma_{i}, \sigma_{j}\right]=\sigma_{i} \sigma_{j}-\sigma_{j} \sigma_{i}=0, \quad i \neq j}
\end{aligned}
$$

Note that two operators from different systems commute. These commutation rules for two-level systems is most commonly found in the literature of the Dicke model [28], that describes a gas of two-level systems emitting in a common radiation field. In the case of a fermion gas, this commutation is an approximation, that is made for the simplicity of the algebra and that is justified for a dilute gas by the fact that anticommuting operators give the same final physical results [29]. Indeed, when the wavefunctions of any two fermions is weakly-overlapping, symmetrized, antisymmetrized and non-symmetrized results are the same [30].

In our case, the two qubits are strongly interacting, which sets our system apart from the Dicke model and its approximations in many respects (see appendix A). For reference, I will analyze the antisymmetrized case of two interacting fermions (Fig. 1d)) completely. However, the main object of interest in this text is that of two commuting qubits (like in the Dicke model), corresponding to the case of two distinguishable (in the quantum sense) qubits (Fig.11(c)).

The level structure of Hamiltonian (1) is sketched in Fig.2(a) (at resonance). Such a "diamond-like" configuration can be mapped to a four-level system (4LS), i.e., as a single entity, with Hilbert space structure $1 \oplus 1 \oplus 1 \oplus 1$ (Fig. 1(e)). This description fits, for instance, the case of a multi-level atom [31] or of a single quantum dot that can host up to two interacting excitons (electron-hole pairs) forming a biexciton state [32]. On the other hand, two coupled qubits, matches the case of two nearby quantum dots directly coupled. The state with double excitation is then called an interdot biexciton state [33].

The Hamiltonian $H_{0}$ can be diagonalized in terms of two 
intermediate dressed states, $|+\rangle$ and $|-\rangle$, as:

$$
H_{0}=\omega_{-}|-\rangle\left\langle-\left|+\omega_{+}\right|+\right\rangle\left\langle+\left|+\omega_{11}\right| 1,1\right\rangle\langle 1,1|
$$

with eigenfrequecies:

$$
\begin{aligned}
& \omega_{ \pm}=\frac{\omega_{1}+\omega_{2}}{2} \pm \mathscr{R}, \quad \mathscr{R}=\sqrt{g^{2}+\left(\frac{\Delta}{2}\right)^{2}}, \\
& \omega_{11}=\omega_{1}+\omega_{2}
\end{aligned}
$$

The diagonalized level structure is sketched in Fig. 2(b). These are the same eigenfrequecies $\omega_{ \pm}$and Rabi splitting (given by $2 \mathscr{R}$ ) than for the dressed states of two coupled harmonic oscillators up to the first manifold [14]. This equivalence breaks in the manifold with two excitations where the fermionic nature of the particles reveals and only the state $|1,1\rangle$ is permitted, as compared to three possible states in the second manifold of the linear model: $\{|2,0\rangle,|1,1\rangle,|0,2\rangle\}$.

The dynamics of dressed states $| \pm\rangle$ and their spectral shape depend on the amount of decoherence that the dissipative and excitation processes induce in the system. I will consider an incoming flow of excitations that populate the two-level systems at rates $P_{1}, P_{2}$ and an outgoing flow (given by the inverse lifetime) at rates $\gamma_{1}, \gamma_{2}$, respectively. This situation corresponds to an incoherent continuous pump or injection in the qubit that can be varied independently from the dissipation. The steady state reached under the pump and decay corresponds to a statistical mixture of all possible quantum states and is described by a density matrix $\rho$. The master equation of the system has the standard Liouvillian form [34], with the corresponding Lindblad terms:

$$
\begin{aligned}
\frac{d \rho}{d t}=\mathscr{L} \rho & =i[\rho, H] \\
& +\sum_{i=1,2} \frac{\gamma_{i}}{2}\left(2 \sigma_{i} \rho \sigma_{i}^{\dagger}-\sigma_{i}^{\dagger} \sigma_{i} \rho-\rho \sigma_{i}^{\dagger} \sigma_{i}\right) \\
& +\sum_{i=1,2} \frac{P_{i}}{2}\left(2 \sigma_{i}^{\dagger} \rho \sigma_{i}-\sigma_{i} \sigma_{i}^{\dagger} \rho-\rho \sigma_{i} \sigma_{i}^{\dagger}\right) .
\end{aligned}
$$

This master equation can be exactly solved given the finite and small dimension of the Hilbert space (which is only four). Within the same formalism, we can describe the spontaneous emission from a general initial state by solving the equations for vanishing pumping.

I will note the transitions between bare states (cf. Fig. 2 (a)) as:

$$
\begin{array}{r}
u_{1}=|0,1\rangle\langle 1,1|=\sigma_{1} \sigma_{2}^{\dagger} \sigma_{2}, \\
l_{1}=|0,0\rangle\langle 1,0|=\sigma_{1}-u_{1}, \\
u_{2}=|1,0\rangle\langle 1,1|=\sigma_{1}^{\dagger} \sigma_{1} \sigma_{2}, \\
l_{2}=|0,0\rangle\langle 0,1|=\sigma_{2}-u_{2} .
\end{array}
$$

(for upper and lower transition). Note that they can all be written in terms of the qubit operators (in normal order) since $\sigma_{i}=u_{i}+l_{i}(i=1,2)$. The Lindblad terms of pump and decay in Eq. (5) are expressed in terms of their $\sigma$ operators. Rewriting them in terms of the transition operators $u_{1,2}, l_{1,2}$ leads to
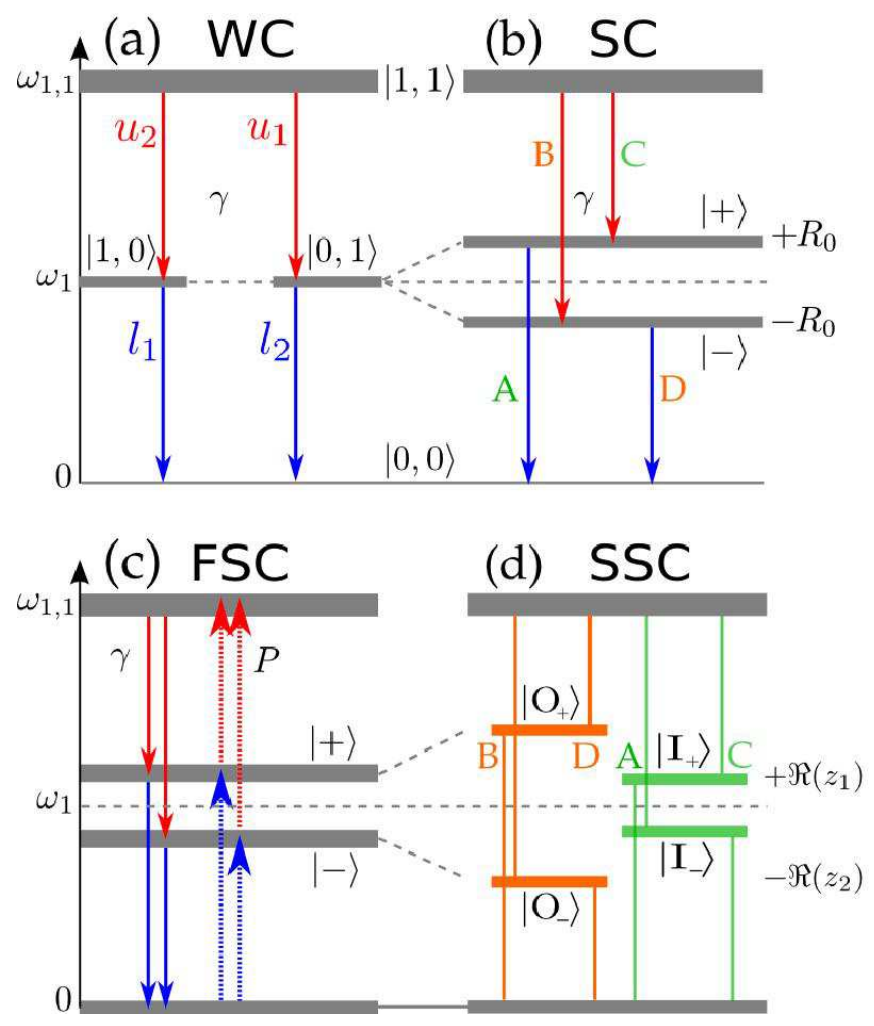

FIG. 2: (Color online) Energy levels for the two qubits described by Hamiltonian (1). Weak coupling (a) and strong coupling (b) in the absence of pump are well defined in terms of the bare and dressed states $| \pm\rangle$, respectively. As shown in Sec. $\mathrm{VB}$ the SC regime gives rise to new regions when pump is taken into account: (c) FSC where dressed states remain $| \pm\rangle$, an (d) SSC an MC, where dressed states form a new set $\left|I_{ \pm}\right\rangle,\left|O_{ \pm}\right\rangle$. Only SSC, with splitting of all the new dressed states, is shown. MC corresponds to the case where $\left|I_{ \pm}\right\rangle$ have closed (both collapsing on $\omega_{1}$ ). The thickness of the levels represents the uncertainty in energy due to $(a, b)$ the decay and (c,d) both the pump and the decay. The arrows linking the levels due to pump/decay are blue for the lower $\left(l_{i}\right)$ and red for the upper $\left(u_{i}\right)$ transitions. Transitions labeled $A, C$ (involving $|+\rangle$ or $\left|\mathrm{I}_{ \pm}\right\rangle$, in green) occur at frequencies determined by $z_{1}$, while $B$ and $D$ (involving $|-\rangle$ or $\left|\mathrm{O}_{ \pm}\right\rangle$, in orange) are determined by $z_{2}$. The same color code is used in the rest of the figures to plot the decomposition of the spectra.

cross Lindblad terms that entangle $l_{1}$ and $u_{1}$, on the one hand, and $l_{2}$ and $u_{2}$ on the other. If the four levels did not correspond to two qubits but to a single entity (a 4LS), such as atomic levels or a single quantum dot levels, the Lindblad terms would be written directly in terms of the transition operators, without these cross Lindblad terms. The alternative master equation is discussed in appendix C(cf. Eq. (C1)).

We introduce fermionic effective broadenings:

$$
\begin{aligned}
& \Gamma_{1}=\gamma_{1}+P_{1}, \quad \Gamma_{2}=\gamma_{2}+P_{2}, \\
& \Gamma_{ \pm}=\frac{\Gamma_{1} \pm \Gamma_{2}}{4}, \quad \gamma_{ \pm}=\frac{\gamma_{1} \pm \gamma_{2}}{4} .
\end{aligned}
$$

Equation (7a) is to be compared with bosonic effective broadenings, for boson modes $a$ and $b$ (such as the harmonic 
oscillators of the linear model):

$$
\tilde{\Gamma}_{a}=\gamma_{a}-P_{a}, \quad \tilde{\Gamma}_{b}=\gamma_{b}-P_{b}
$$

A tilde is being used to denote the bosonic character of the broadening, in the sense that the pumping strength is subtracted to the decay. Pumping leads to broadening of the line in the fermionic case and narrowing in the bosonic case, which is a spectral manifestation of Fermi and Bose statistics.

Another convenient notation for Eqs. (7) is the expression of pumping and decay rates in terms of $\Gamma$ s and a new parameter $r$ which represents the type of reservoir that the qubit is in contact with [35]:

$$
\begin{aligned}
& \gamma_{1}=\Gamma_{1}\left(1-r_{1}\right), \quad \gamma_{2}=\Gamma_{2}\left(1-r_{2}\right), \\
& P_{1}=\Gamma_{1} r_{1}, \quad P_{2}=\Gamma_{2} r_{2} .
\end{aligned}
$$

All possible situations (with $0 \leq r_{i} \leq 1$ ), from a medium that only absorbs excitation, $r_{i}=0$, to one which only provides them, $r_{i}=1$, are thus included in a transparent way. For instance, a thermal bath with temperature different from zero corresponds to $r_{i}<1 / 2$. The effect of the medium on the effective broadening is contained in $\Gamma_{i}$.

The power spectrum for each qubit, $i=1,2$, is defined as:

$s_{i}(\omega)=\left\langle\sigma_{i}^{\dagger}(\omega) \sigma_{i}(\omega)\right\rangle=\frac{1}{2 \pi} \Re \int_{0}^{\infty} \int_{0}^{\infty} G_{i}^{(1)}(t, t+\tau) e^{i \omega \tau} d t d \tau$

where

$$
G_{i}^{(1)}(t, t+\tau)=\left\langle\sigma_{i}^{\dagger}(t) \sigma_{i}(t+\tau)\right\rangle
$$

is the first order auto correlation function. $s(\omega)$ describes how energy is distributed and is thus of fundamental interest. In a quantum optical context, this can be observed directly in the optical emission, but for generality, I will keep the terminology of power spectrum. For the steady state spectrum, the running time $t$ is taken at infinite values (thereby removing one integral). In appendix B, we make use of the quantum regression formula [34] in its most general form to compute $G_{i}^{(1)}$ as well as other two- and one-time correlators (second order correlation functions are given in appendix E). We shall focus on $i=1$ in the following, without loss of generality:

$$
G_{1}^{(1)}(t, t+\tau)=n_{1} \sum_{p=A, B, C, D}\left[L_{p}(t)+i K_{p}(t)\right] e^{-i \omega_{p} \tau} e^{-\frac{\gamma_{p}}{2} \tau},
$$

where $n_{1}=\int s_{1}(\omega) d \omega$, the population of qubit 1 , is used to normalize the expression for the spectrum (so that $\left.\int S_{1}(\omega) d \omega=1\right)$ :

$$
\begin{aligned}
S_{1}(\omega)=\frac{1}{\pi} \sum_{p \in\{A, B, C, D\}}\left[L_{p} \frac{\frac{\gamma_{p}}{2}}{\left(\frac{\gamma_{p}}{2}\right)^{2}+\left(\omega-\omega_{p}\right)^{2}}\right. \\
\\
\left.\quad-K_{p} \frac{\omega-\omega_{p}}{\left(\frac{\gamma_{p}}{2}\right)^{2}+\left(\omega-\omega_{p}\right)^{2}}\right],
\end{aligned}
$$

from Eqs. 10. and 12.
The spectrum is composed of four peaks that I label $p=$ $A, B, C, D$, each of them with a Lorentzian (weighted by the coefficient $L_{p}$ ) and a dispersive part (weighted by $K_{p}$ ). The four resonant frequencies $\omega_{p}$ and the associated broadenings (full-widths at half maximum) $\gamma_{p}$, are intrinsic to the system, as they correspond to the four possible transitions in the system. The coefficients $K_{p}$ and $L_{p}$ (derived in appendix $\mathrm{B}$ ) are the parameters that are specific to the experimental configuration (such as channel of detection) or regime (steady state under incoherent pumping or spontaneous emission of an initial state). This form of $S(\omega)$ is a general feature for the power spectra of coupled quantum systems [36].

In the case of uncoupled qubits $(g=0)$, the four resonances reduce to the two bare energies $\omega_{1}$ and $\omega_{2}$, broadened by the effective decay rates $\Gamma_{1}$ and $\Gamma_{2}$, respectively. The peaks are, in this case, pure Lorentzians. In the opposite case of very strong coupling $(g \gg \gamma, P)$, the spectrum is also well approximated by Lorentzians, but with the resonances $\omega_{p}$ now at the dressed state frequencies $\omega_{ \pm}$, and broadened by the average rates $\left(\Gamma_{1}+\Gamma_{2}\right) / 2$. Lorentzian lineshapes correspond to the emission of well defined isolated modes of the system Hamiltonian, weakly affected by other modes. In this case, $K_{p} \approx 0$. The dispersive contribution becomes non negligible in the intermediate situations when dissipation and decoherence (or dephasing, cf. appendix A are of the order of the direct coupling. The Hamiltonian eigenmodes are then no longer neatly leading the dynamics and, as a consequence, their broad emission lines overlap in energy, producing interferences. This is the regime of interest in our analysis, since new phenomenology appears for the dressed states and coupling regimes. With this goal in mind, I devote the next two sections to presenting the analytical expressions of all the quantities appearing in the spectrum, Eq. 113.

\section{SINGLE-TIME DYNAMICS}

We start by analyzing the relevant average quantities needed to compute the weights $L_{p}$ and $K_{p}$ in the spectrum (13), that is, populations and coherences:

$$
\begin{aligned}
& n_{1}=\left\langle\sigma_{1}^{\dagger} \sigma_{1}\right\rangle, \quad n_{2}=\left\langle\sigma_{2}^{\dagger} \sigma_{2}\right\rangle \in \mathbb{R}, \\
& n_{\text {corr }}=\left\langle\sigma_{1}^{\dagger} \sigma_{2}\right\rangle \in \mathbb{C}, \\
& n_{11}=\left\langle\sigma_{1}^{\dagger} \sigma_{1} \sigma_{2}^{\dagger} \sigma_{2}\right\rangle \in \mathbb{R} .
\end{aligned}
$$

$n_{i}$ is the probability that qubit $i$ is excited. The sum $n_{1}+n_{2}$, that can go up to two, is the total excitation in the system. $n_{\text {corr }}$ is the effective coherence between the qubits due to the direct coupling. $n_{11}$ is the joint probability that both qubits are excited. It is also the population of state $|1,1\rangle$. If the qubits were uncoupled, we would have $n_{11}=n_{1} n_{2}$.

It is important here to outline the difference between population of a mode (say, $n_{1}=\left\langle\sigma_{1}^{\dagger} \sigma_{1}\right\rangle$ for a qubit and $n_{a}=\left\langle a^{\dagger} a\right\rangle$ for an harmonic oscillator, cf. Fig. 1(a) and (c)), and population of a state $\left(\rho_{10}\right.$ and $\tilde{\rho}_{10}$ for the state $|1,0\rangle$ with $|1,0\rangle=$ $a^{\dagger}|0,0\rangle$ and $\sigma_{1}^{\dagger}|0,0\rangle$, respectively). The population of the intermediate state $|1,0\rangle$ (resp. $|0,1\rangle$ ) is given by $n_{1}-n_{11}$ 
(resp. $n_{2}-n_{11}$ ). This is also the probability of having only one of the qubits excited. The population of the ground state is given by $1-n_{1}-n_{2}+2 n_{11}$.

In the spontaneous emission case, $n_{1}, n_{2}$ and $n_{\text {corr }}$ have the same solutions (depending only on the initial condition $n_{1}^{0}$, $n_{2}^{0}$ and $n_{\text {corr }}^{0}$ ) than their counterpart for the two coupled linear oscillators, $n_{a}, n_{b}$ and $n_{\mathrm{ab}}$. However, the two models differ for $n_{11}$ : in the case of coupled qubits, it decays from its initial value, $n_{11}(t)=e^{-4 \gamma_{+} t} n_{11}^{0}$, whereas in the linear model, the population $\tilde{\rho}_{11}$ oscillates as a result of the exchange with the other states that are available in the second manifold $(|2,0\rangle$ and $|0,2\rangle$ ). Although populations of the modes have the same dynamics both in the two harmonic oscillators and the two coupled qubits, the underlying populations of their states do not. For instance, the decay from the initial condition $|1,1\rangle$ leads to $n_{1}=n_{10}+n_{11}=\rho_{10}+\rho_{11}$ for the qubit, while for the harmonic oscillators, one has, $n_{a}=\left\langle a^{\dagger} a\right\rangle=\tilde{\rho}_{10}+\tilde{\rho}_{11}+2 \tilde{\rho}_{20}$. Since $n_{1}=n_{a}$ (and also $n_{2}=n_{b}$ ) the states are differently populated $\left(\rho_{10} \neq \tilde{\rho}_{10}\right.$, Etc. $)$.

In the steady state, all these mean values can be written in terms of effective pump and decay parameters, as in the two harmonic oscillators, but now following fermionic statistics $(i=1,2)$ :

$$
\begin{aligned}
n_{i}^{\mathrm{SS}} & =\frac{P_{i}^{\mathrm{eff}}}{\gamma_{i}^{\mathrm{eff}}+P_{i}^{\mathrm{eff}}}, \\
\gamma_{i}^{\mathrm{eff}} & =\gamma_{i}+\frac{\gamma_{1}+\gamma_{2}}{\Gamma_{1}+\Gamma_{2}} Q_{i}, \quad P_{i}^{\mathrm{eff}}=P_{i}+\frac{P_{1}+P_{2}}{\Gamma_{1}+\Gamma_{2}} Q_{i}, \\
n_{\mathrm{corr}}^{\mathrm{SS}} & =\frac{g}{\Delta-2 i \Gamma_{+}}\left(n_{1}-n_{2}\right),
\end{aligned}
$$

with the corresponding generalized Purcell rates

$$
Q_{1}=\frac{4\left(g^{\mathrm{eff}}\right)^{2}}{\Gamma_{2}}, \quad Q_{2}=\frac{4\left(g^{\mathrm{eff}}\right)^{2}}{\Gamma_{1}}
$$

and the effective coupling strength

$$
g^{\mathrm{eff}}=\frac{g}{\sqrt{1+\left(\frac{\Delta / 2}{\Gamma_{+}}\right)^{2}}}
$$

Finally, $n_{11}$ takes a simple intuitive form in the steady state,

$$
n_{11}^{\mathrm{SS}}=\frac{n_{1}^{\mathrm{SS}} P_{2}+n_{2}^{\mathrm{SS}} P_{1}}{\Gamma_{1}+\Gamma_{2}} \leq n_{1}^{\mathrm{SS}} n_{2}^{\mathrm{SS}} .
$$

This should be contrasted with the counterpart of $n_{11}^{\text {SS }}$ for two bosonic modes ( $a$ and $b$ ), for which:

$$
\left\langle a^{\dagger} a b^{\dagger} b\right\rangle^{\mathrm{SS}}=2 n_{a}^{\mathrm{SS}} n_{b}^{\mathrm{SS}}-\frac{n_{a}^{\mathrm{SS}} P_{b}+n_{b}^{\mathrm{SS}} P_{a}}{\Gamma_{a}+\Gamma_{b}} \geq n_{a}^{\mathrm{SS}} n_{b}^{\mathrm{SS}} .
$$

This is an interesting manifestation of the symmetry/antisymmetry of the wavefunction for two bosons/fermions, that is known to produce such an attractive/repulsive character for the correlators. Here we see that quantum (or correlated) averages $\left\langle\hat{n}_{1} \hat{n}_{2}\right\rangle$ (with $\hat{n}_{i}$ the number operator) are higher/smaller than classical (uncorrelated) averages $\left\langle\hat{n}_{1}\right\rangle\left\langle\hat{n}_{2}\right\rangle$, depending on whether they are

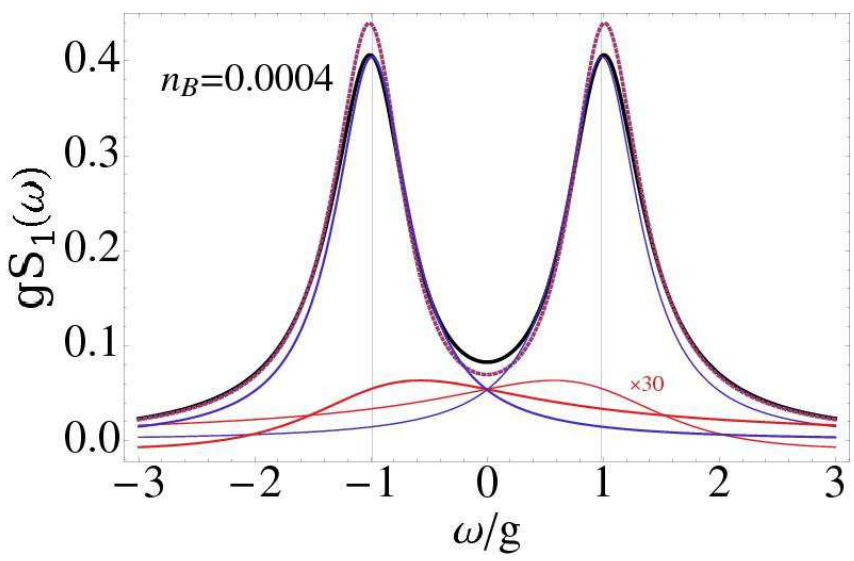

FIG. 3: (Color online) Power spectrum, $S_{1}(\omega)$, from a qubit (thick solid black line) in the SC regime $\left(\gamma_{1}=g\right.$ and $\left.\gamma_{2}=g / 2\right)$ for the steady state under vanishing pump $\left(P_{1}=0.02 g\right.$ and $\left.P_{2}=0.01 g\right)$. The spectra is composed of four peaks arising from the lower and upper transitions (blue and red thin lines, respectively). In dashed purple, the linear model spectrum for comparison.

of a boson or fermion character, respectively. This provides a neat picture of bunching/antibunching from excitations of different modes that are otherwise of the same character.

In the most general case, with pump and decay, before the steady state is reached, also the transient dynamics of the mean values $n_{1}, n_{2}$ and $n_{\text {corr }}$ for the coupled qubits maps to the corresponding averages $n_{a}, n_{b}$ and $n_{a b}$ of coupled harmonic oscillators, with only $\tilde{\Gamma} \rightarrow \Gamma$. We can conclude then, that the single-time dynamics of the qubit is ruled by a (half) Rabi frequency of the same form than in the boson case [14]:

$$
R^{1 \mathrm{TD}}=\sqrt{g^{2}-\left(\Gamma_{-}+i \Delta / 2\right)^{2}}
$$

only with Fermion-like effective broadenings, Eqs. (7). Since, in contrast to the boson case where there is only one Rabi parameter, another expression will arise in the two-time dynamics for coupled qubits, I will refer to Eq. (20) as the single-time dynamics (half) Rabi frequency.

We conclude this section by noting that the magnitudes studied up to now are independent on having two qubits, two identical fermions or a 4LS. The cross terms appearing in the master equation for the first case, due to the correlations induced by the incoherent processes, do not affect the steady state populations, as is shown in appendix $\mathrm{C}$

\section{POWER SPECTRA}

\section{A. Two qubits}

The general expressions for the spectrum and two-time correlators of two coupled qubits admit analytic solutions at resonance in the steady state of an incoherent continuous pump. From now on, we will refer always to this situation and, therefore, I will drop the steady state label in the notation. 
The four coefficients $L_{p}+i K_{p}$ appearing in Eq. (12), now defined in the steady state, read: (cf. appendix B)

$$
\begin{aligned}
L_{A}+i K_{A}= & \frac{1}{16 R z_{1}}\left\{2\left(2 z_{1}+i \Gamma_{2}\right)\left(R-i \Gamma_{-}\right)+a_{1}+a_{2} \frac{n_{2}}{n_{1}}\right. \\
& \left.+2 g\left[-\frac{P_{1}}{\Gamma_{+}}\left(2 z_{1}+i \Gamma_{2}\right)+2\left(R+z_{1}+i \Gamma_{+}\right)\right] \frac{n_{\text {corr }}}{n_{1}}\right\},
\end{aligned}
$$

$$
\begin{aligned}
L_{B}+i K_{B}= & \frac{1}{16 R z_{2}}\left\{2\left(2 z_{2}+i \Gamma_{2}\right)\left(R+i \Gamma_{-}\right)-a_{1}-a_{2} \frac{n_{2}}{n_{1}}\right. \\
& \left.+2 g\left[\frac{P_{1}}{\Gamma_{+}}\left(2 z_{2}+i \Gamma_{2}\right)+2\left(R-z_{2}-i \Gamma_{+}\right)\right] \frac{n_{\text {corr }}}{n_{1}}\right\},
\end{aligned}
$$

$$
\begin{aligned}
L_{C}+i K_{C}= & \frac{1}{16 R z_{1}}\left\{2\left(2 z_{1}-i \Gamma_{2}\right)\left(R-i \Gamma_{-}\right)-a_{1}-a_{2} \frac{n_{2}}{n_{1}}\right. \\
& \left.+2 g\left[-\frac{P_{1}}{\Gamma_{+}}\left(2 z_{1}-i \Gamma_{2}\right)-2\left(R-z_{1}+i \Gamma_{+}\right)\right] \frac{n_{\text {corr }}}{n_{1}}\right\},
\end{aligned}
$$

$$
\begin{aligned}
L_{D}+i K_{D}= & \frac{1}{16 R z_{2}}\left\{2\left(2 z_{2}-i \Gamma_{2}\right)\left(R+i \Gamma_{-}\right)+a_{1}+a_{2} \frac{n_{2}}{n_{1}}\right. \\
& \left.+2 g\left[\frac{P_{1}}{\Gamma_{+}}\left(2 z_{2}-i \Gamma_{2}\right)-2\left(R+z_{2}-i \Gamma_{+}\right)\right] \frac{n_{\text {corr }}}{n_{1}}\right\} .
\end{aligned}
$$

They are defined in terms of the parameters

$a_{1}=\frac{g^{2}}{\Gamma_{+}^{2}}\left[4 \Gamma_{+}^{2}+2 P_{1}\left(P_{2}-2 \Gamma_{+}\right)-P_{2} \Gamma_{1}\right], \quad a_{2}=\frac{g^{2}}{\Gamma_{+}^{2}} P_{1}\left(P_{1}-\gamma_{1}\right)$,

and the corresponding frequencies and decay rates, that also appear explicitly in Eq. (12):

$$
\begin{array}{ll}
\frac{\gamma_{A}}{2}+i \omega_{A}=2 \Gamma_{+}+i z_{1}, & \frac{\gamma_{B}}{2}+i \omega_{B}=2 \Gamma_{+}+i z_{2}, \\
\frac{\gamma_{C}}{2}+i \omega_{C}=2 \Gamma_{+}-i z_{1}, & \frac{\gamma_{D}}{2}+i \omega_{D}=2 \Gamma_{+}-i z_{2} .
\end{array}
$$

They all depend on two complex parameters, $z_{1}$ and $z_{2}$ :

$$
z_{1,2}=\sqrt{\left(D^{\mathrm{s}} g\right)^{2}+\left(i \Gamma_{+} \pm R\right)^{2}} .
$$

The degree of symmetry, $D^{\mathrm{s}}$, is a real dimensionless quantity, between 0 and $\sqrt{2}$, given by

$$
D^{\mathrm{s}}=\frac{\sqrt{\left(\gamma_{1} P_{2}+\gamma_{2} P_{1}\right) / 2}}{\Gamma_{+}} .
$$

This quantity is proper to the coupled qubits case and its physical meaning will be clarified later. Its value is linked to the symmetry between the different parameters. For instance, $D^{\mathrm{s}}=1$ when all parameters are equal to each other, $\gamma_{1}=\gamma_{2}=P_{1}=P_{2}$. On the other hand, $D^{\mathrm{s}}=0$ if one of the parameters (any of them) is much larger than the others. It leads to a renormalized coupling strength

$$
G=D^{\mathrm{s}} g,
$$

that reaches a maximum when the parameters are such that $D^{\mathrm{s}}=\sqrt{2}$. Such an enhancement, by $\sqrt{2}$, is related to the cooperative behavior of two coupled modes, similarly to the superradiance of two atoms in the Dicke model or the renormalization with the mean number of photons in the JaynesCummings Model.

The last and most important parameter appearing in the previous expressions is a Rabi frequency for the two-time dynamics, that for coupled qubits differs from its counterpart for single-time dynamics (cf. Eq. 20):

$$
R=\sqrt{g^{2}-\left(D^{\mathrm{s}} g\right)^{2}-\Gamma_{-}^{2}} .
$$

This is the true analog of the (half) Rabi frequency of the linear model since this value, not its single-time counterpart, determines strong or weak coupling (emergence of dressed states). At vanishing pump, the renormalized coupling $G$ converges to $g$, and both $R$ and $R^{1 \mathrm{TD}}$ converge to the standard expression for the (half) Rabi splitting [14]:

$$
R_{0}=\sqrt{g^{2}-\gamma_{-}^{2}} .
$$

The normalized power spectrum of qubit 1 follows from Eq. (13) with the coefficients we have obtained. The positions and broadenings of the four peaks are given respectively by the real and imaginary parts of $z_{1}$ and $z_{2}$. Their expressions remain valid in the spontaneous emission case by setting the pumping rates to zero.

Figure 3 is an example of the spectrum $S_{1}(\omega)$ (in solid black) and its decomposition in four peaks (thin blue and red). The split positions of the four peaks, which indicate the system is in the SC regime, are marked with two vertical blue lines. The two peaks that correspond to the lower manifold transitions, $A$ and $D$ in Fig. 2(b), appear with a thin blue line. Upper transitions, $B$ and $C$ in Fig. 2(b), appear with a thin red line. All resonances are at the same positions but the stronger dispersive part of upper transitions leads to a shift of their maximum. The upper transitions are much weaker in intensity (magnified $\times 30$ to be visible) due to the small pump. The double excitation of the system is very unlikely $\left(n_{11}=0.0004\right)$. The lineshape is therefore close to that of two coupled harmonic oscillators, plotted with a dashed purple line for comparison. The system is in the linear regime where all models of Fig. 1 for two coupled modes converge. In the following sections, we will see how the lineshapes change when entering the nonlinear regime.

\section{B. Two fermions}

As noted before, although the expressions for the singletime dynamics (populations, coherence, Etc.) for two coupled fermions (anticommuting operators for modes 1 and 2) are the same than for the two coupled qubits, their power spectra are different. As compared to the coupled qubits, the coupled fermions spectrum assumes a simple and fundamental form, closely related to that of the two harmonic oscillators: the formal expression is the same, differing only in the parameters (effective broadenings, populations, Etc.). In particular, only 
one Rabi parameter, the single-time Rabi frequency, $R^{1 \mathrm{TD}}$, determines both the single- and two-time dynamics. The twofermions spectra are thus obtained by simply substituting the fermionic parameters (Eq. 7) in the expression of the linear model [14].

This simplicity and likeliness to the linear model stems from the fundamental nature of the problem: two identical (indistinguishable) particles coupled linearly, obeying fully their quantum statistics. The two coupled qubits (or the JaynesCummings model [16]), by mixing different types of particles (distinguishable modes) and therefore breaking commutation rules, result in the more complex description and richer dynamics presented in the previous section.

\section{C. four-level system (4LS)}

Also in the four-level system (with no cross Lindblad terms), the expressions for the single-time dynamics (populations, coherence, Etc.) are the same than for the linear model, and here also their power spectra are different. The parameters for the 4LS spectra are of a bosonic character, cf. Eq. (8):

$$
\begin{aligned}
& \tilde{\Gamma}_{1}=\gamma_{1}-P_{1}, \quad \tilde{\Gamma}_{2}=\gamma_{2}-P_{2}, \\
& \tilde{\Gamma}_{ \pm}=\frac{\tilde{\Gamma}_{1} \pm \tilde{\Gamma}_{2}}{4}, \\
& \tilde{R}=\sqrt{g^{2}-\tilde{\Gamma}_{-}^{2}} .
\end{aligned}
$$

The relevant parameters that characterize the coupling simplify to:

$$
\begin{aligned}
& R=\frac{\tilde{\Gamma}_{+}}{\Gamma_{+}} \tilde{R}, \\
& z_{1,2}=\tilde{R} \pm i \tilde{\Gamma}_{+},
\end{aligned}
$$

recovering the conventional strong coupling criterion based on one parameter only, the bosonic (half) Rabi frequency $\tilde{R}$. The resulting spectral structure then consists of two pairs of peaks sitting at $\pm \Re(\tilde{R})$ with:

$$
\begin{aligned}
& \frac{\gamma_{A}}{2}+i \omega_{A}=\frac{3\left(P_{1}+P_{2}\right)+\gamma_{1}+\gamma_{2}}{4}+i \tilde{R}, \\
& \frac{\gamma_{B}}{2}+i \omega_{B}=\frac{3\left(\gamma_{1}+\gamma_{2}\right)+P_{1}+P_{2}}{4}+i \tilde{R}, \\
& \frac{\gamma_{C}}{2}+i \omega_{C}=\frac{3\left(\gamma_{1}+\gamma_{2}\right)+P_{1}+P_{2}}{4}-i \tilde{R}, \\
& \frac{\gamma_{D}}{2}+i \omega_{D}=\frac{3\left(P_{1}+P_{2}\right)+\gamma_{1}+\gamma_{2}}{4}-i \tilde{R} .
\end{aligned}
$$

Note that $\gamma_{p}$ are always positive for any combination of the parameters, in contrast with those of two bosonic modes, where the system can diverge. Therefore, the values of pump and decay rates here are not limited, always leading to a physical steady state.

\section{STRONG AND WEAK COUPLING REGIMES}

The standard criterion for strong coupling (SC) is based on the splitting at resonance of the bare states into dressed states. This manifests in the appearance of $\tau$-oscillations in the twotime correlators and a splitting of the peaks that compose their spectrum.

In a naive approach to the problem of defining strongcoupling in a system other than the linear model, one could think that the condition for $\mathrm{SC}$ is $\Re\left(R^{1 \mathrm{TD}}\right) \neq 0$ (at resonance), leading to the familiar inequality, $g>\left|\Gamma_{-}\right|$. However, this is not the case whenever pump and decay are both taken into account. Instead, one must find the condition for a splitting between the new eigenstates, that is, the two pairs of peaks forming the spectrum. The peaks are positioned symmetrically in two pairs about the origin at $\omega_{p}= \pm \Re\left(z_{1,2}\right)$ and, therefore,

$$
\mathfrak{R}\left(z_{1}\right) \neq 0 \text { or } \mathfrak{R}\left(z_{2}\right) \neq 0
$$

is the mathematical condition for SC in this system. Given that there are two different parameters $z_{1}$ and $z_{2}$ on which the condition relies, the SC/WC distinction must be extended to cover new possibilities. Thus, instead of only one relevant parameter, $\Gamma_{-} / g$, as was the case in the linear model, SC between two qubits is determined by three parameters:

$$
\Gamma_{-} / g, \quad \Gamma_{+} / g \text { and } D^{\mathrm{s}} \text {. }
$$

This gives rise to the situations listed in Table \, that are discussed in the following sections.

\begin{tabular}{|c|c|c|c|c|}
\hline$R$ & $\Re\left(z_{1}\right)$ & $\mathfrak{R}\left(z_{2}\right)$ & Acronym & Type of coupling \\
\hline \hline$|R|$ & $\neq 0$ & $\neq 0$ & FSC & First order Strong Coupling \\
$i|R|$ & $\neq 0$ & $\neq 0$ & SSC & Second order Strong Coupling \\
$i|R|$ & 0 & $\neq 0$ & MC & Mixed Coupling \\
$i|R|$ & 0 & 0 & WC & Weak Coupling \\
\hline
\end{tabular}

TABLE I: Type and nomenclature of coupling for two coupled qubits. Beyond the usual weak coupling (WC) and strong coupling (here denoted FSC) encountered in the linear model, the system exhibits two new regions: Mixed Coupling (coexistence of weak and strong coupling) and Second order Strong Coupling (with two different splittings of two pairs of dressed states).

\section{A. Vanishing pump and spontaneous emission}

In the case of vanishing pump, that corresponds as well to spontaneous emission, the standard SC and WC hold. In this limit, we recover the familiar expression for the half Rabi frequency $R, R^{1 \mathrm{TD}} \rightarrow R_{0}$. The parameters simplify to $z_{1,2} \rightarrow \sqrt{\left(R_{0} \pm i \gamma_{+}\right)^{2}}=R_{0} \pm i \gamma_{+}[39]$.

The positions and broadenings of the four peaks are:

$$
\begin{array}{lll}
\frac{\gamma_{A}}{2}+i \omega_{A}=\gamma_{+}+i R_{0}, & \frac{\gamma_{B}}{2}+i \omega_{B}=3 \gamma_{+}+i R_{0}, \\
\frac{\gamma_{C}}{2}+i \omega_{C}=3 \gamma_{+}-i R_{0}, & \frac{\gamma_{D}}{2}+i \omega_{D}=\gamma_{+}-i R_{0} .
\end{array}
$$


From here, the associated condition for SC reduces to $R_{0}$ being real, or more explicitly $g>\left|\gamma_{-}\right|$, as in the linear model at vanishing pump [see Fig. 2(b)]. In SC, the two pairs of peaks $p=A, D$ and $p=B, C$ sit on the same frequencies although they have different broadenings. Excited states have shorter lifetime, since each excitation can decay. From Eqs. (34), the two dressed states undergo the transition into weak coupling (WC) simultaneously [as in Fig. 2[a)]. In WC, $R_{0} \rightarrow i\left|R_{0}\right|$ and both parameters $z_{1,2}$ become imaginary, giving

$$
\begin{aligned}
& \omega_{p}=0, \quad p=A, B, C, D, \\
& \frac{\gamma_{A}}{2}=\gamma_{+}-\left|R_{0}\right|, \quad \frac{\gamma_{B}}{2}=3 \gamma_{+}-\left|R_{0}\right|, \\
& \frac{\gamma_{C}}{2}=3 \gamma_{+}+\left|R_{0}\right|, \quad \frac{\gamma_{D}}{2}=\gamma_{+}+\left|R_{0}\right|,
\end{aligned}
$$

with $\gamma_{p} \geq 0$, since $\gamma_{+} \geq\left|R_{0}\right|$ in this regime. The four peaks collapse into four Lorentzians at the origin, all differing in their broadenings.

As a result of the two pairs of peaks sitting always on the same two (or one) frequencies, the final spectra can only be either a single peak or a doublet, both shapes being possible in SC or WC regimes (as in the linear model and for the same reasons [14]). An intuitive derivation and interpretation of these results is given in appendix $\mathrm{D}$, based on the so-called manifold picture [13], which consists in considering transitions between eigenstates of a non-hermitian Hamiltonian, with energies broadened by the imaginary part.

In the steady state case but in the limit of vanishing pump (the linear regime), only the vacuum and first manifold are populated. The spectra in this limit converge with the linear model and also it can be analyzed in terms of manifolds by straightforward extension. The spectrum in Fig. (3) is an example of SC for vanishing pump as we can see from the fact that the lower transition peaks, in blue, dominate over the broader and weak upper peaks, in red. In this case, the splitting of the dressed modes gives rise to a splitting in the final spectrum (in black). In what follows, we take this SC configuration $\left(\gamma_{1}=g\right.$ and $\left.\gamma_{2}=g / 2\right)$ as a starting point to explore the effect of a non-negligible incoherent continuous pump.

\section{B. Non-negligible pump}

When pump is taken into account, all the types of coupling listed in Table \are accessible. These are plotted in Fig. (4) as a function of the pumping rates. The starting point is the Rabi frequency $R$, Eq. [27), that is either real or pure imaginary.

First, let us consider the case where:

$$
R=|R| \quad \Leftrightarrow \quad G^{2}<g^{2}-\Gamma_{-}^{2},
$$

from which follows that $z_{1}=z_{2}^{*}$, and therefore $\Re\left(z_{1}\right)=\Re\left(z_{2}\right)$. This is the most standard situation that we already found in the absence of pumping. It is sketched in Fig. 2(c). To distinguish it from the other types of coupling to be discussed shortly, I will from now on call it First order Strong Coupling (FSC). Note that condition (36) can only be satisfied if $D^{\mathrm{s}}<1$, therefore, when the renormalization of the coupling through

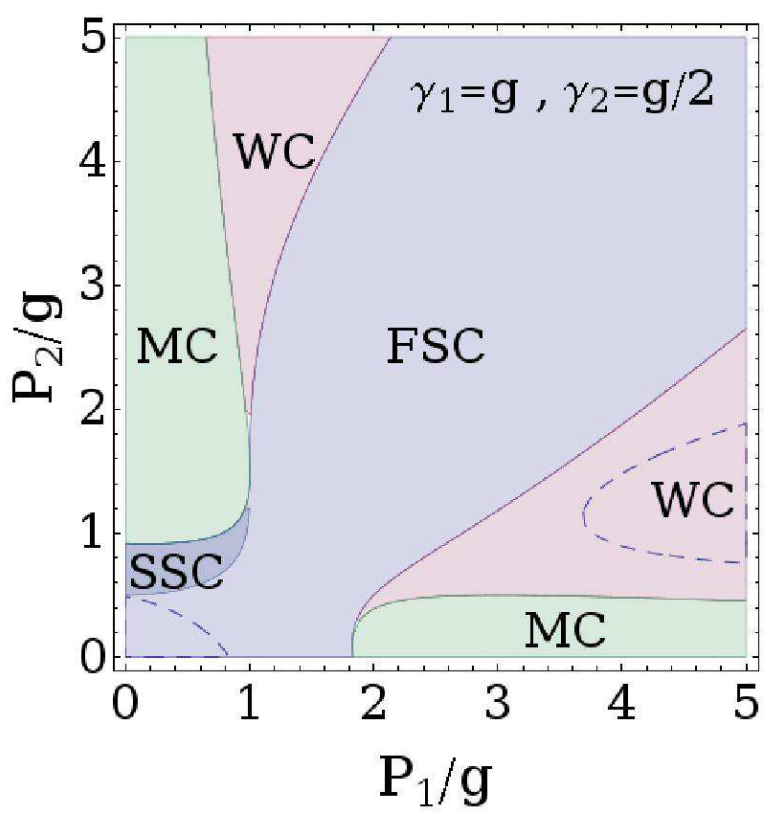

FIG. 4: (Color online) Phase space of the steady state Strong/Weak Coupling regimes as a function of pump for $\gamma_{1}=g$ and $\gamma_{2}=g / 2$. In Strong Coupling (SC, blue), one can distinguish two regions, First order (FSC, light blue) and Second order (SSC, dark blue) Strong Coupling. Weak Coupling (WC) is in purple and Mixed Coupling (MC) in green. The dashed blue lines enclose the two regions where two peaks can be resolved in the power spectrum of the first qubit, $S_{1}(\omega)$. One falls in SC and the other in WC.

the interplay of pump and decay is detrimental (since Eq (36) implies that $G<g$ ). It is not possible to reach the optimum effective coupling and maximum splitting of the spectral lines given by $2 \sqrt{2} g$. Eq. (36) leads to the following explicit condition for $g$ :

$$
g>\frac{\left|\Gamma_{-}\right|}{\sqrt{\left|1-\left(D^{\mathrm{s}}\right)^{2}\right|}} .
$$

If $R \neq 0$, then, also $\Re\left(z_{1}\right)=\Re\left(z_{2}\right) \neq 0$. FSC includes the standard SC regime in the absence of pump $\left(g>\left|\Gamma_{-}\right|\right.$that is implied by Eq. (37)). It is the most extended region in Fig. 4, colored in light blue. In this case, the spectrum of emission follows the expected pattern: two pairs of peaks, $A, D$ and $B, C$, are placed one on top of each other, although they are differently broadened [see the spectra in Fig. 3].

In Fig. 5 (a) and (b) we track the broadenings and positions of the four peaks ( $A$ and $D$ in blue and $B$ and $C$ in red) as a function of pump, through the SC region of Fig 4 , on a diagonal line defined by $P_{2}=P_{1} / 2$. Following them from vanishing pump, where the manifold picture is exact, the four peaks can be easily associated with the lower and upper transitions of Fig. 2(b), and that is why we keep the same color code and notation. Dressed states $| \pm\rangle$, close to the Hamiltonian ones, can still be defined in the system, but with the modified frequencies $\omega_{1} \pm \Re\left(z_{1}\right)$, both affected equally by decoherence.

By construction, the resulting spectra in this regime can only be a doublet or a single peak, depending on the mag- 
nitude of the broadening of the peaks (that always increases with pump and decay) against the splitting of the lines (that always decreases). As in the limit of vanishing pump, observing a doublet in the spectra does not imply splitting of the dressed states (and thus, SC) [37], but here the tendency is always the same: the lower the pump and the decay, the better the resolution of the splitting.

Second, let us consider the situation of the Rabi frequency being imaginary:

$$
R=i|R| \quad \Leftrightarrow \quad G^{2}>g^{2}-\Gamma_{-}^{2} .
$$

This results in three possibilities, listed in Table @(WC, SSC and $\mathrm{MC}$ ), that constitute the three remaining regions delimited in Fig. 4. In what follows we find the specificities of each of these three regimes. by

The Weak Coupling regime (WC, in purple) is characterized

$$
z_{1}=i\left|z_{1}\right|, \quad z_{2}=i\left|z_{2}\right|, \quad z_{1} \neq z_{2} \quad \Leftrightarrow \quad G<\left|\Gamma_{+}-\right| R||
$$

and therefore $\mathfrak{R}\left(z_{1}\right)=\mathfrak{R}\left(z_{2}\right)=0$. Note that condition 39 ) is not analytical in terms of the relevant parameters (33). In $\mathrm{WC}$, the four peaks are placed at the origin with four different broadenings. The dressed states have collapsed in energy to $\omega_{1}$.

Up to here, we have remained within the SC and WC regions already known from the linear model. We now consider the two new regions of SC, proper to the coupled qubits, that I call SSC and MC, respectively:

SSC: When both parameters $z_{1,2}$ are real, then:

$$
z_{1}=\left|z_{1}\right|, \quad z_{2}=\left|z_{2}\right|, \quad z_{1}<z_{2} \quad \Leftrightarrow \quad G>\left|\Gamma_{+}+\right| R|| .
$$

We refer to it as Second order Strong Coupling regime (SSC, colored in dark blue in the phase space). Here, the broadenings of the four peaks are equal, $\gamma_{p} / 2=2 \Gamma_{+}$, but the positions of the pairs of peaks are different, $\omega_{A, C}= \pm\left|z_{1}\right|$ and $\omega_{B, D}= \pm\left|z_{2}\right|$. The reason is that the bare energies of the modes undergo a second order anticrossing induced by the interplay between coupling, pump and decay. The energies of the dressed states are affected differently by decoherence, up to the point where we may picture the physics in terms of a new type of eigenstates. The association of the $A$ and $D$ (with $\left.\omega_{A, D}\right)$ as the peaks corresponding to lower transitions and $B$ and $C$ (with $\omega_{B, C}$ ) to upper transitions is completely arbitrary in this region, given that the broadenings of the peaks, which led us to such association in FSC, are now equal. This implies that, rather than two dressed states, $|-\rangle$ and $|+\rangle$ (Fig. 2 (b)) as in the conventional strong coupling (FSC), the system now exhibits four dressed states: $\left|I_{-}\right\rangle,\left|I_{+}\right\rangle,\left|O_{-}\right\rangle$and $\left|O_{+}\right\rangle$. They are plotted in Fig. 22(d): $\left|\mathrm{I}_{ \pm}\right\rangle$(resp. $\left|\mathrm{O}_{ \pm}\right\rangle$) have energies split at $\pm\left|z_{1}\right|$, giving rise to the inner peaks, in green (resp. $\pm\left|z_{2}\right|$, giving rise to the outer peaks, in orange). The physical origin of this remarkable departure from the conventional strong coupling picture will be discussed in section VII.

We can see how peak broadenings and positions change when going from FSC to SSC in Fig. 5 (c) and (d). In this case, we track the peaks by varying $P_{2}$ for a fixed $P_{1}$, moving upwards in the phase space. The first vertical guideline

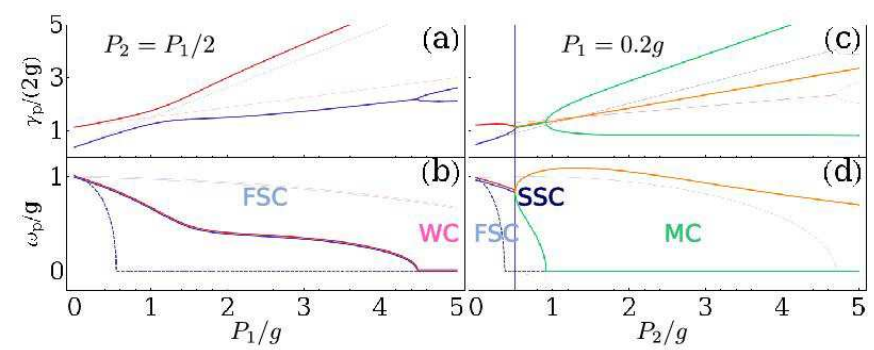

FIG. 5: (Color online) Broadenings (a), (c), and positions (b), (d) of the lines that compose the spectra as a function of pump for the decay parameters $\gamma_{1}=g$ and $\gamma_{2}=g / 2$. In the plots of the first column, the pump $P_{1}$ varies with $P_{2}=P_{1} / 2$, moving upwards in the phase space of Fig 4 The vertical guideline shows the crossing from FSC to WC. In the plots of the second column, the pump $P_{2}$ varies with $P_{1}=0.2 g$, moving in diagonal in the phase space of Fig 4 The vertical guidelines show the crossing from FSC to SSC and finally to MC. The dashed blue line represents the splitting as it is resolved in the final spectrum $S_{1}(\omega)$. The color code (red-blue and orangegreen) corresponds to that of the transitions in Fig. 2

marks the border between the two kinds of SC, with the opening of a "bubble" for the positions $\omega_{A}$ and $\omega_{B}$ (that were equal in the FSC region), and the convergence of all the broadenings. In principle, one can expect that quadruplets and triplets may form out of the four peaks. However, the broadenings and contributions of the dispersive parts (given by $K_{p}$ ) are too large to let any fine splitting emerge clearly. The spectra in this region reduce to singlets and doublets. However, we show in Sec.VIthrough some examples that they may be distorted, doubtlessly reflecting the multiplet structure.

$M C$ : When $z_{1}$ is imaginary and $z_{2}$ real, or equivalently,

$$
z_{1}=i\left|z_{1}\right|, \quad z_{2}=\left|z_{2}\right| \quad \Leftrightarrow \quad\left|\Gamma_{+}-\right| R||<G<\left|\Gamma_{+}+\right| R||,
$$

we enter the last new region in Fig. 4. This is a Mixed Coupling regime (MC, colored in green in the phase space) where the two inner peaks, $\mathrm{A}$ and $\mathrm{C}$-as well as the reconstructed eigenstate $\left|\mathrm{I}_{ \pm}\right\rangle$-have collapsed at the origin, like in WC. However, the two outer peaks, B and D-as well as $\left|\mathrm{O}_{ \pm}\right\rangle$are still split. As in SSC, the broadening of the peaks does not allow for a distinction between upper of lower resonances. The collapsed resonance is in this case at the bare energy $\omega_{1}=0$ because that is the total average bare resonance in the system. In Sec. VIA we will see that when the qubits are detuned, this resonance happens at $\left(\omega_{1}+\omega_{2}\right) / 2=-\Delta / 2$.

Again, although one may expect a triplet in $\mathrm{MC}$, only distorted singlets are observed in the best of cases due to the broadening and dispersive parts. In Fig. 5(c) and (d) we can see the transition from SSC into MC, at the second vertical line.

Note that, in this system, the pumping mechanism is equivalent to an upward decay, due to the ultimate saturation of the qubit and the symmetry in the schema of levels that they form. The master equation is symmetrical under exchange of the pump and the decay $\left(\gamma_{i} \leftrightarrow P_{i}\right)$ when the two-levels of both qubit are inverted $(|0,0\rangle \leftrightarrow|1,1\rangle$ and $|1,0\rangle \leftrightarrow|0,1\rangle)$ [40]. Consequently, the parameters $z_{1}, z_{2}$ and $R$, and also the popu- 
lations of all the levels, are symmetric in the same way, as it happens with just one qubit. In other systems, like the linear model, the Jayne-Cummings model or simply a single harmonic oscillator, the effect of the pump extends upwards to an infinite number of manifolds while the decay cannot bring the system lower than the ground state. There is no natural truncation for the pump (that ultimately leads to a divergence), as there is for the decay. But with coupled qubit, state $|1,1\rangle$ is the upper counterpart of $|0,0\rangle$, undergoing a saturation. This implies, for instance, that the (transient) dynamics in the limit of vanishing decay is exactly the same as that of vanishing pump and that in such case we can also apply the manifold method to obtain the right positions and broadenings as a function of pump, in the same way that we did as a function of decay only. We only have to take into account the mentioned symmetry consistently. As long as the dynamics moves upwards or downwards only, even when intermediate states are coupled, the manifold picture is suitable. The manifold diagonalization breaks, however, in the presence of both nonnegligible pump and decay. This is discussed in appendix D.

\section{PARTICULAR CASES}

In this section, we illustrate the rather abstract previous discussions with examples. The symmetry in the decay and pumping rates determines the effective coupling, emission properties and dressed states. Let us start by expressing $D^{\mathrm{s}}$, the magnitude quantifying such symmetry, in terms of the reservoir parameters $\left(\Gamma_{i}\right.$ and $\left.r_{i}\right)$,

$$
D^{\mathrm{s}}=\sqrt{2} \frac{\sqrt{\Gamma_{1} \Gamma_{2}}}{\left(\Gamma_{1}+\Gamma_{2}\right) / 2} \sqrt{r_{1}+r_{2}-2 r_{1} r_{2}} .
$$

In this form, its physical meaning is more clear. There are two separate factors to discuss: the symmetry in the strength of the couplings to the reservoirs, given by $\sqrt{\Gamma_{1} \Gamma_{2}} /\left[\left(\Gamma_{1}+\Gamma_{2}\right) / 2\right]$ and plotted in Fig. 6 (a), and the symmetry in the nature of the reservoirs given by $\sqrt{r_{1}+r_{2}-2 r_{1} r_{2}}$, plotted in Fig. 6 b).

The coupling $g$ is enhanced when

$$
g<G \leq \sqrt{2} g, \quad \text { that is, } \quad 1<D^{\mathrm{s}} \leq \sqrt{2},
$$

which happens when $r_{1}>1 / 2$ and $r_{2}<1 / 2$ (or the other way around). This corresponds to the two squared regions with lighter colors in Fig. 6 b). Then, the two reservoirs are of opposite natures: the reservoir of the first qubit provides excitations $\left(P_{1}>\gamma_{1}\right)$ while the other absorbs them $\left(P_{2}<\gamma_{2}\right)$. If this is accompanied by similar interaction strengths, $\Gamma_{1} \sim \Gamma_{2}$, enhancement occurs. We refer to these situations as optimally pumped and study them in Sec.VIA

On the other hand, if the reservoirs are of the similar natures, both $r_{1}, r_{2} \geq 1 / 2$ or $\leq 1 / 2$, both providing or absorbing particles, then the system is detrimentally pumped:

$$
0 \leq G \leq g, \quad \text { that is, } \quad 0 \leq D^{\mathrm{s}} \leq 1
$$

It corresponds to the two squared regions with darker colors in Fig. 6. (b). We study this in Sec.VIB.
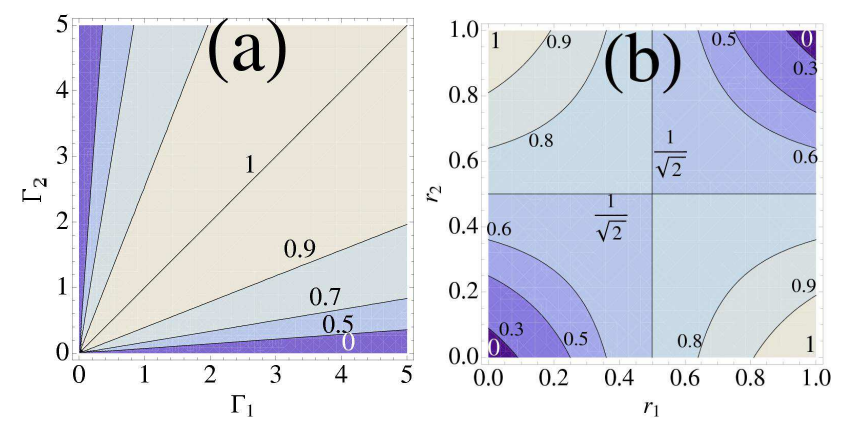

FIG. 6: Factors contributing to $D^{\mathrm{s}}$ : (a) $\sqrt{\Gamma_{1} \Gamma_{2}} /\left[\left(\Gamma_{1}+\Gamma_{2}\right) / 2\right]$ as a function of $\Gamma_{1}, \Gamma_{2}$ and (b) $\sqrt{r_{1}+r_{2}-2 r_{1} r_{2}}$ as a function of $r_{1}, r_{2}$. The values corresponding to the contour lines are marked on the plots. Both functions take values from 0 (dark blue) to 1 (light).

\section{A. Optimally pumped cases: $g<G \leq \sqrt{2} g$}

Let us explore the optimally pumped cases by considering parameters on the diagonal $\Gamma_{1}=\Gamma_{2}$ in Fig. 6) a) together with the antidiagonal $r_{1}+r_{2}=1$ in Fig. 6(b). The reservoirs have opposite nature but interact with equal strength with the qubits. This corresponds to the situation where the decay and pumping parameters are equal in a crossed way:

$$
P_{1}=\gamma_{2}, \quad \text { and } \quad P_{2}=\gamma_{1} \text {. }
$$

The system has a total input that is equal to the total output, $P_{\text {TOT }}=P_{1}+P_{2}=\gamma_{\text {TOT }}=\gamma_{1}+\gamma_{2}$, and also equal Purcell rates, $Q_{1}=Q_{2}$. The excited and ground states are formally equivalent in the dynamics.

Figure 7 shows the different coupling regimes accessible with this configuration, as a function of $P_{1}$ and $P_{2}$ with the same color code than in Fig. 4. This configuration is in FSC only when all parameters are equal, $D^{\mathrm{S}}=1$, (blue line) and there is total symmetry in the system. Otherwise, one of the new type of coupling (SSC in blue or MC in green) is realized as the coupling is effectively improved, $G>g$. In the inset the type of spectral shapes that results is shown.

\begin{tabular}{|c|c|c|}
\hline Lineshape & $L_{\mathrm{sl}}$ & $L_{\mathrm{con}}$ \\
\hline \hline singlet & 1 & 2 \\
distorted singlet & 1 & 6 \\
doublet & 3 & 4 \\
distorted doublet & 3 & 8 \\
triplet & 5 & 6 \\
quadruplet & 7 & 8 \\
\hline
\end{tabular}

TABLE II: The lineshapes $S_{1}(\omega)$ are defined by two quantities: $L_{\mathrm{sl}}$ is the number of times that $S_{1}(\omega)$ changes slope, that is, the number of real solutions to the equation $d S_{1}(\omega) / d \omega=0 ; L_{\mathrm{con}}$ is the number of times that $S_{1}(\omega)$ changes concavity, that is, the number of real solutions to the equation $d^{2} S_{1}(\omega) / d \omega^{2}=0$.

The vertical axis in Fig.7 with $P_{1}=\gamma_{2}=\gamma$ and $P_{2}=\gamma_{1}=0$, is illustrative of all the possible coupling regions and lineshapes. This is the extreme situation of optimal pumping where the two reservoirs interact equally strongly with the 


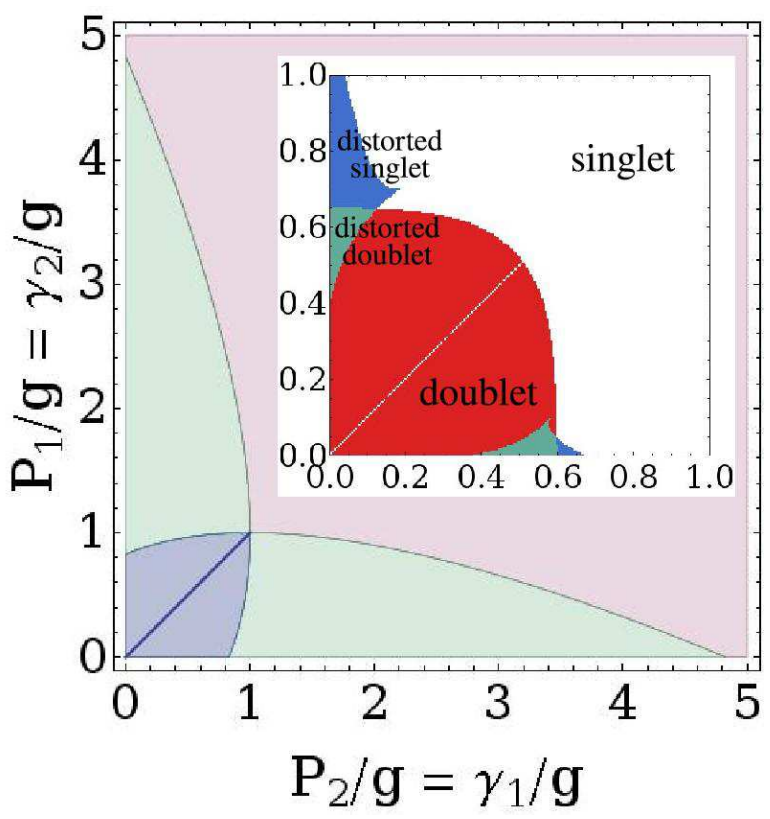

FIG. 7: (Color online) Phase space of FSC/SSC/MC/WC as function of $P_{1} / g=\gamma_{2} / g$ and $P_{2} / g=\gamma_{1} / g$. The color code is that of Fig.4 In inset, the possible lineshapes of $S_{1}(\omega)$ : a doublet (red), a distorted doublet (green), a distorted singlet (blue) and a singlet (white), as explained in Table

two qubits $\left(\Gamma_{1}=\Gamma_{2}=\gamma\right)$ and have completely opposite natures: one only providing particles $\left(r_{1}=1\right)$ and the other only absorbing them $\left(r_{2}=0\right)$. At this point, there is maximum renormalization of the coupling, $G=\sqrt{2} g\left(D^{\mathrm{s}}=\sqrt{2}\right)$ as both factors in Eq. (42) are maximum. The populations and mean values read:

$$
\begin{aligned}
& n_{2}=\frac{2}{4+(\gamma / g)^{2}}, \quad n_{1}=1-n_{2} \\
& n_{11}=\frac{1}{4+(\gamma / g)^{2}} \neq n_{1} n_{2} \\
& n_{\text {corr }}=-i \frac{\gamma / g}{4+(\gamma / g)^{2}} .
\end{aligned}
$$

The two qubits share one excitation only. The Rabi frequency also simplifies to $R=i g$ (as $\left.\Gamma_{-}=0\right)$, and

$$
z_{1,2}=\sqrt{g^{2}-(\gamma / 2)^{2} \mp g \gamma}
$$

In Fig. 8, we can see some of these magnitudes varying in the different regimes as a function of $\gamma / g$. In the linear regime limit, $\gamma \ll g$, there is FSC with all the levels equally populated $\left(n_{1}=n_{2}=1 / 2, n_{11}=1 / 4\right)$ and $n_{\text {corr }}=-i(\gamma / g) / 4$. As soon as there is pumping, the SSC opens a "bubble" in the eigenenergies with the splitting of inner and outer peaks. The transition into $\mathrm{MC}$, with the collapse of the inner peaks, takes place at $\gamma=2(\sqrt{2}-1) g$, and the transition into WC, closing the bubble, takes place at $\gamma=2(\sqrt{2}+1) g$. The maximum of $z_{2}=\sqrt{2} g$ (in orange) takes place at $\gamma=2 g$, when the coherence $\left|n_{\text {corr }}\right|=1 / 4$ is maximum. This is a special point where
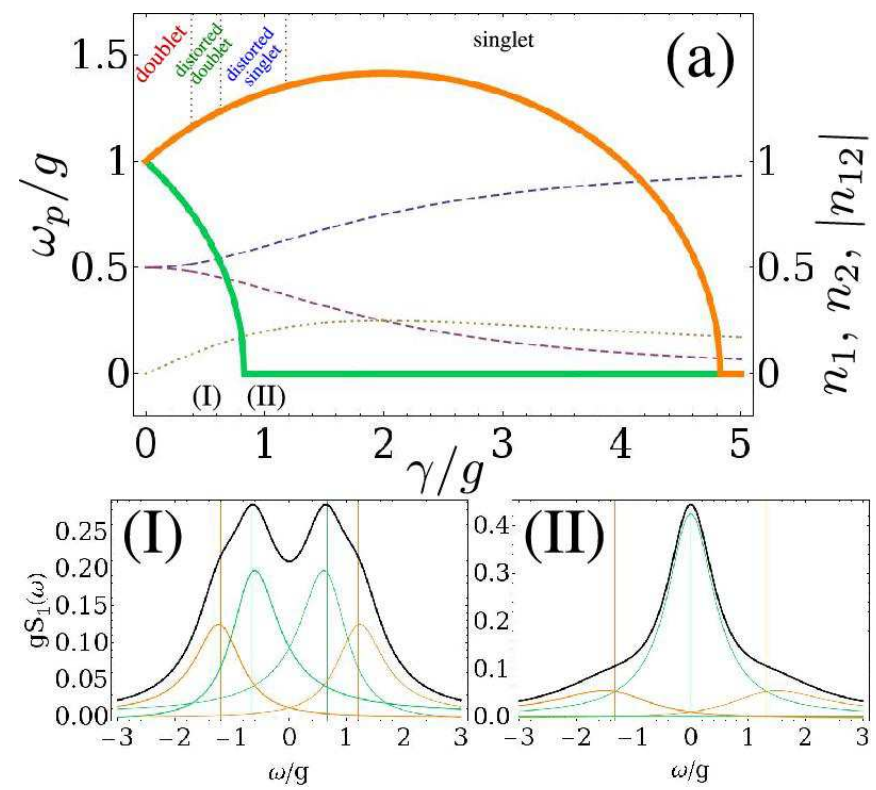

FIG. 8: (Color online) (a) Positions of the peaks $\left(\omega_{A}\right.$ in thick green, $\omega_{B}$ in thick orange) and populations $\left(n_{1}\right.$ in dashed blue, $n_{2}$ in dashed purple, $\left|n_{\text {corr }}\right|$ in dotted brown) as function of $P_{1} / g=\gamma_{2} / g=\gamma / g$, for $P_{2}=\gamma_{1}=0$. The system goes from FSC (at 0) to SSC, to MC, to WC, while the lineshape of the spectra changes as coded in Table I The most interesting lineshapes, that can only appear in SSC and MC, are the distorted doublet (I) and singlet (II). The total spectra (in black) is decomposed in inner (green) and outer (orange) peaks coming from the transitions sketched in Fig.2 d).

the splitting of the dressed modes is the largest possible, $2 \sqrt{2} g$ (even though the lineshape remains a singlet). Finally, when the coupling becomes very weak, $\gamma \gg g$, the first dot saturates, quenching the exchange of excitation between the qubit and $n_{2}=n_{11}=n_{\text {corr }}=0$.

Although the underlying physics of coupling is very rich and complex, the spectra do not acquire distinctively marked lineshapes (such as well resolved triplets of quadruplets): a doublet in SSC only gets distorted, but this is unambiguously due to the underlying quadruplet structure, as shown in Fig. [8 I), and also a singlet gets distorted due to the underlying triplet structure, in (II). Before reaching WC, the spectrum has become a plain singlet. The way to distinguish mathematically the different possible shapes and their origin in underlying triplets and quadruplets is by counting zeros of first and second derivatives, as given in Table II of which only the four first lines are realized in the coupled qubit.

In Fig. 9 we make the comparison between the results discussed in Fig. 8 and those obtained for a 4LS (without cross Lindblad terms), where the system undergoes a standard transition SC-WC as $\gamma$ increases. From Sec. IVC, we know that, although the populations do not change from those in Eqs. (46), the position of the four peaks differ, as well as the coupling regimes. In this case the parameters $z$ converge to:

$$
z_{1,2} \rightarrow \sqrt{g^{2}-(\gamma / 2)^{2}}
$$

This corresponds to a splitting intermediate between those of 

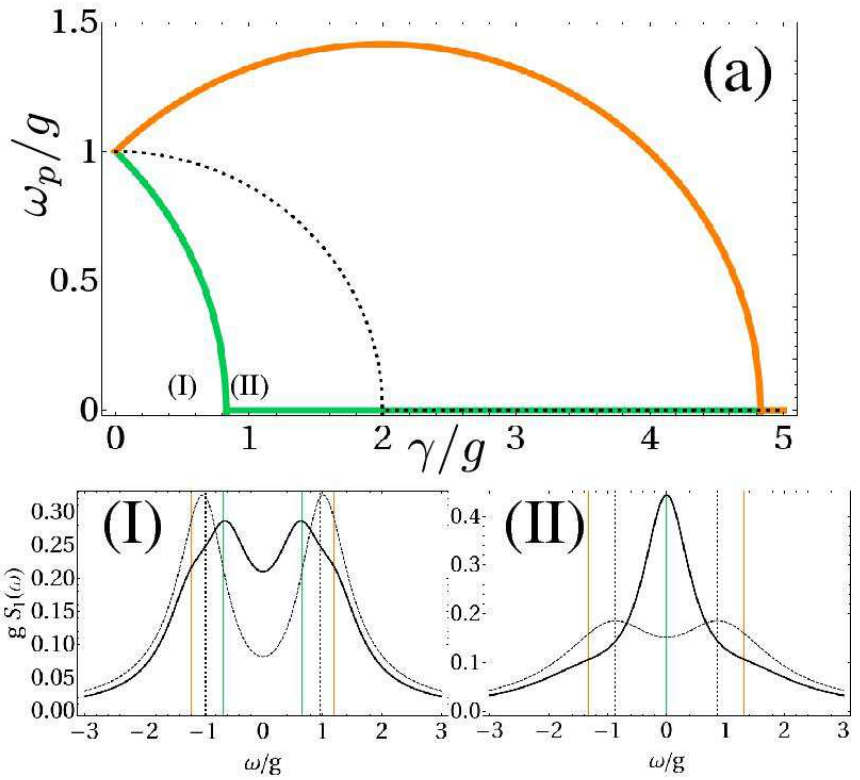

FIG. 9: (Color online) Comparison between the results of Fig. 8 (solid lines) and those obtained for a 4LS (dashed black lines). The positions of the peaks composing the spectra, are marked with vertical lines in (I) and (II). In a 4LS, only plain doublets and singlets arise. The populations are the same for both cases. Different types of splitting lead to dramatic differences in the spectral shapes.

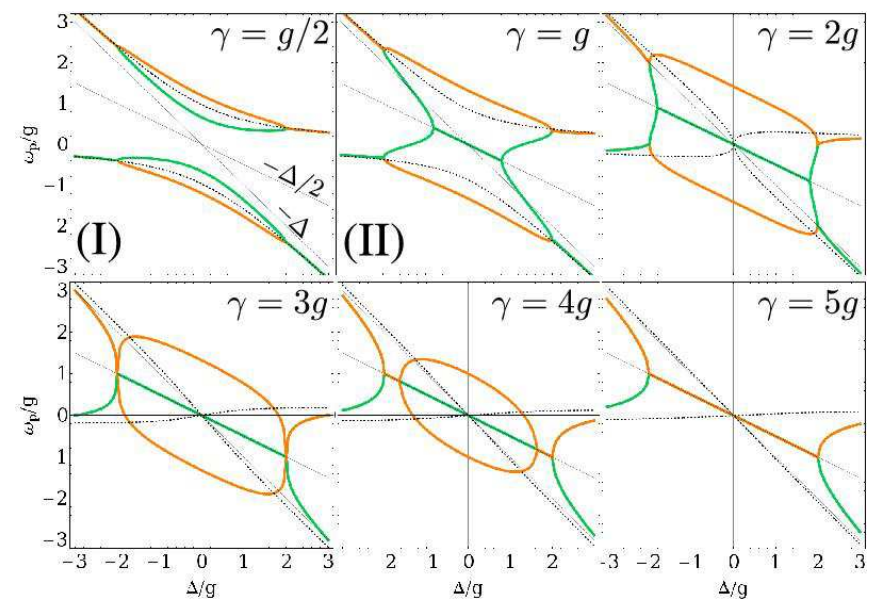

FIG. 10: (Color online) (a) Positions of the peaks $\left(\omega_{A, C}\right.$ in thick green, $\omega_{B, D}$ in thick orange) as a function of detuning for six cases in Fig. 8 with the specified parameter $\gamma$. The positions in the absence of crossed terms appear in dashed black and in the absence of coupling in thin black, for comparison. The first two plots are those labeled (I) and (II) in previous Figures.

the inner and outer peaks, as we can see in dashed black in Fig.9a). Although the 4LS splitting is smaller than the splitting between outer peaks (in orange), the SC doublet in the spectrum is better resolved than in the case of two qubit, due to the large intensity of the inner peaks.

Fig. 10 displays the behaviors of all the resonances as a function of detuning for six cases in Fig. 8 (see insets), including (I) $\gamma=g / 2$ and (II) $\gamma=g$. Inner and outer peaks
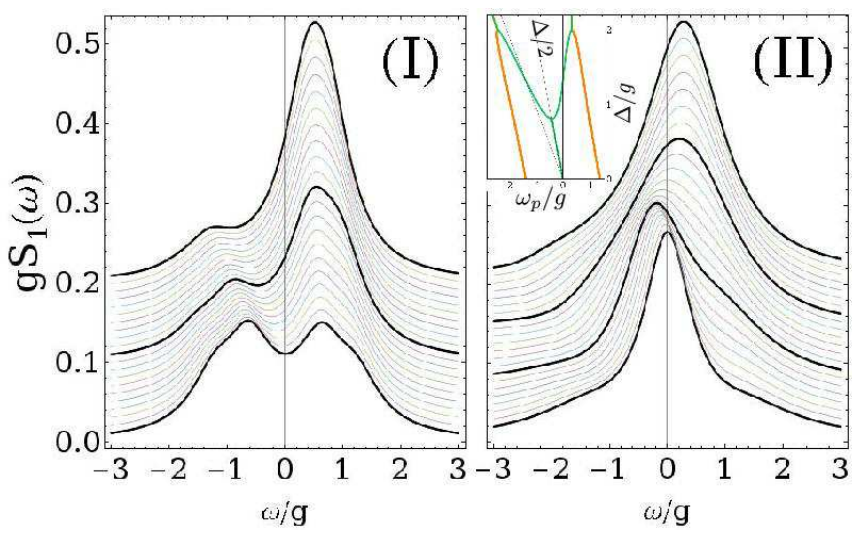

FIG. 11: (Color online) Spectra for varying positive detuning (anticrossings) in SSC (I) and in MC (II) for the corresponding cases in Fig. 9 The inset in (II) shows the positions of the three/four peaks composing the spectra as a function of detuning. Close to resonance, the inner peaks converge to the average transition energy $\omega_{11} / 2=\Delta / 2$. At large detunings, the first dot (that is pumped) emits at $\omega_{1}=0$ and dominates over the second dot (that decays) with emission at $\omega_{2}=-\Delta$.

superimpose whenever there are only one or two resonances. Otherwise, green lines represent inner resonances and orange lines the outer ones. Dashed black lines represent the case of a 4LS, which simply splits in two lines close to resonance in SC (the transition to WC happens at $\gamma=2 g$ ). In thin black, the bare energies $\left(\omega_{1}=0, \omega_{2}=-\Delta\right)$ are plotted as a reference, and recovered in all cases very far from resonance or well into WC. The 4LS resonances converge to the bare energies at all detunings almost as soon as they enter WC. However, the resonances of two qubits, when closing in WC (MC in the case of inner resonances), collapse to the average between the bare energies $\left(\omega_{1}+\omega_{2}\right) / 2=-\Delta / 2$ instead. At resonance these two behaviors are equivalent, but at small detunings they are clearly very different. We will discuss the reason for this puzzling saturation at $-\Delta / 2$ instead of $-\Delta$ in Sec. VII Eventually, at very large $\gamma$, the bare energies will be recovered both by inner and outer peaks.

The anticrossing that the lineshapes form when detuning between the modes is varied from zero to some detuning $\Delta_{\max }$, is also peculiar. In Fig. 11 (I) and (II) we can see that the distorted doublet and singlet keep their features up to $\Delta_{\max }=g$ and $\Delta_{\max }=2 g$ (resp.). The resonances in Fig. 11 are not equally present in the emission. In both cases, at large detuning, the emission at $\omega_{1}=0$ of the first qubit is dominant over that of the second qubit at $\omega_{2}=-\Delta$ because the first qubit is being pumped and the second only dissipates the excitation. Note that, the singlet in (II) slightly oscillates to the left before joining the origin at large detuning. This is a clear signature that the central peak is not simply a feature of $\mathrm{WC}$, but of a more complicated MC eigenstate structure, as can be seen in the inset. This leftwards shift is a consequence of the splittings saturating at $-\Delta / 2$ instead of the bare energies $(0$ and $-\Delta)$. This shift is even more evident when both inner and outer peaks saturate at some detuning, like in case $\gamma=4 g$. 


\section{B. Detrimentally pumped cases: $0<G \leq g$}

To explore the detrimentally pumped cases, we consider parameters on the diagonal $r_{1}=r_{2}=r$ in Fig. 6(b), where the reservoirs have the same nature.

If the reservoirs also have the same interaction strength with the qubit, $\Gamma_{1}=\Gamma_{2}$, the two qubits become indistinguishable. This corresponds to equal dissipation and pump,

$$
\gamma_{1}=\gamma_{2}=\gamma \quad \text { and } \quad P_{1}=P_{2}=P
$$

The symmetry in the system is not total as the incomeoutcome flows of excitation may not be balanced, $\gamma_{\text {TOт }} \neq$ $P_{\text {TOT. }}$. The steady state populations are the same as for the uncoupled case with $n_{1}=n_{2}=P /(\gamma+P), n_{11}=n_{1} n_{2}, n_{\text {corr }}=0$, as happens with any two indistinguishable coupled modes (like, for instance, harmonic oscillators). This does not mean that the qubit are uncoupled, in fact, the system can be considered always in strong coupling (FSC) with

$$
\begin{aligned}
& R=\frac{|\gamma-P|}{\gamma+P} g=2\left|r-\frac{1}{2}\right| g, \\
& D^{\mathrm{S}}=\frac{\sqrt{\gamma P}}{(\gamma+P) / 2}=2 \sqrt{r(1-r)} .
\end{aligned}
$$

If $P \gg \gamma(r=1)$, or the other way around $(r=0)$, the symmetry between the flows (inwards and outwards) is completely broken and $D^{\mathrm{S}}=0$, although, at the same time, the splitting in dressed modes is maximum $R=g$ (although not enhanced).

On the other hand, if $P=\gamma(r=1 / 2)$, the symmetry is total $\left(D^{\mathrm{S}}=1\right)$. Here, all the levels are equally populated $\left(n_{1}=n_{2}=\right.$ $\left.1 / 2, n_{11}=1 / 4\right)$ and the Rabi frequency vanishes $R=0$. The SC/WC regimes become conventional with $z_{1,2}=\sqrt{g^{2}-\gamma^{2}}$. The SC condition is simply given by $g>\gamma$. This particular situation is depicted in Fig. 7 with a thick dark blue line in SC that goes into WC when the parameters equal 1.

A second possibility that results in degrading the effective coupling, still considering reservoirs of the same nature $\left(r_{1}=\right.$ $r_{2}=r$ ), is when $r=1 / 2$, which means:

$$
\gamma_{1}=P_{1}=\Gamma_{1} / 2 \text { and } \gamma_{2}=P_{2}=\Gamma_{2} / 2 .
$$

Although the parameters may be different, $\Gamma_{1} \neq \Gamma_{2}$, there is compensation between the flows (inwards and outwards) $\gamma_{\text {TОТ }}=P_{\text {TOT }}$. This configuration arises when both qubits are in a thermal bath of infinite temperature. The populations are also those of the uncoupled system, $n_{1}=n_{2}=1 / 2$, $n_{11}=n_{1} n_{2}, n_{\text {corr }}=0$. The Rabi and degree of symmetry read

$$
R=\frac{\left|\Gamma_{-}\right|}{\Gamma_{+}} \sqrt{g^{2}-\Gamma_{+}^{2}} \text { and } D^{\mathrm{s}}=\frac{\sqrt{\Gamma_{1} \Gamma_{2}}}{\left(\Gamma_{1}+\Gamma_{2}\right) / 2} .
$$

The FSC condition reads now $g>\Gamma_{+}$.

\section{DRESSED STATES}

Now that we have described the new regimes appearing in this system due to the incoherent pumping, SSC and MC, and their spectral features, we can discuss their origin.
We have pointed out that the manifold picture, based on the Hamiltonian dynamics, breaks in presence of non-negligible pumping. To define dressed states in such an essentially nonHamiltonian system, one must recourse to another formalism, presented in this section, based on studying the flow of coherence in the system. This allows a reconstruction of dressed states that recovers the manifold picture for vanishing pump and extends it otherwise.

To analyze how coherence flows in the system, let us address the dynamics of coherence between states, given by offdiagonal elements of the density matrix $\rho$. We thus consider the vector of the relevant transitions:

$$
\mathbf{v}_{\mathrm{coh}}=\left(\begin{array}{c}
\left\langle u_{1}\right\rangle \\
\left\langle l_{1}\right\rangle \\
\left\langle u_{2}\right\rangle \\
\left\langle l_{2}\right\rangle
\end{array}\right)=\left(\begin{array}{c}
\langle 1,1|\rho| 0,1\rangle \\
\langle 1,0|\rho| 0,0\rangle \\
\langle 1,1|\rho| 1,0\rangle \\
\langle 0,1|\rho| 0,0\rangle
\end{array}\right) .
$$

The dynamics of this vector is ruled by a matrix $\mathbf{M}_{\mathrm{coh}}$ :

$$
d \mathbf{v}_{\mathrm{coh}} / d t=-\mathbf{M}_{\mathrm{coh}} \mathbf{v}_{\mathrm{coh}}
$$

that reads:

$$
\mathbf{M}_{\mathrm{coh}}=\left(\begin{array}{cccc}
\frac{\Gamma_{1}}{2}+\gamma_{2} & -P_{2} & -i g & 0 \\
-\gamma_{2} & \frac{\Gamma_{1}}{2}+P_{2} & 0 & i g \\
-i g & 0 & \frac{\Gamma_{2}}{2}+\gamma_{1}-i \Delta & -P_{1} \\
0 & i g & -\gamma_{1} & \frac{\Gamma_{2}}{2}+P_{1}-i \Delta
\end{array}\right) .
$$

The diagonal terms in $\mathbf{M}_{\text {coh }}$ give the decay rate of each coherence and the off-diagonal terms, the rates at which coherences is transferred from one transition to another. Its eigenvalues recover the positions and broadenings of the spectrum peaks in Eq. 23, with eigenstates that I note $\left\langle\mathrm{T}_{p}\right\rangle, p=A, B, C, D$. Therefore, the single time dynamics of $\left\langle\mathrm{T}_{p}\right\rangle$ is free:

$$
\left\langle\mathrm{T}_{p}(t)\right\rangle=e^{-\left(i \omega_{p}+\gamma_{p} / 2\right)\left(t-t_{0}\right)}\left\langle\mathrm{T}_{p}\left(t_{0}\right)\right\rangle,
$$

and their spectral shape is a Lorentzian:

$$
s_{T_{p}}(\omega)=\left\langle\mathrm{T}_{p}^{\dagger}(\omega) \mathrm{T}_{p}(\omega)\right\rangle=\frac{\left\langle\mathrm{T}_{p}^{\dagger} \mathrm{T}_{p}\right\rangle}{\pi} \frac{\gamma_{p} / 2}{\left(\gamma_{p} / 2\right)^{2}+\left(\omega-\omega_{p}\right)^{2}}
$$

In this way, one can reconstruct the dressed states from the above expressions by inspection of the $\left\langle T_{p}\right\rangle$.

First, let us consider the simplest case of Hamiltonian dynamics (without pumping and decay), for which we already obtained the dressed states through the manifold picture (by diagonalization of the Hamiltonian with only direct coupling $g$ ). The following discussion will thus reconstruct Fig. 22 (b). The eigenenergy $\omega_{A}=\omega_{B}=+i g$ corresponds to the two eigenstates:

$$
\begin{array}{ll}
\left\langle\mathrm{T}_{A}\right\rangle=\left\langle d_{1}+d_{2}\right\rangle & \text { (lower transition }|0,0\rangle \leftrightarrow|+\rangle), \\
\left\langle\mathrm{T}_{B}\right\rangle=\left\langle u_{2}-u_{1}\right\rangle \quad & \text { (upper transition }|-\rangle \leftrightarrow|1,1\rangle) .
\end{array}
$$

The eigenenergy $\omega_{C}=\omega_{D}=-i g$ corresponds to the counterpart eigenstates:

$$
\begin{aligned}
& \left.\left\langle\mathrm{T}_{C}\right\rangle=\left\langle d_{1}-d_{2}\right\rangle \quad \text { (transition }|0,0\rangle \leftrightarrow|-\rangle\right), \\
& \left\langle\mathrm{T}_{D}\right\rangle=\left\langle u_{2}+u_{1}\right\rangle \quad(\text { transition }|+\rangle \leftrightarrow|1,1\rangle) .
\end{aligned}
$$


Therefore, the system resonances $\left\langle T_{p}\right\rangle$ give away the existence of the dressed states:

$$
| \pm\rangle \propto \pm|1,0\rangle+|0,1\rangle
$$

(left not normalized for convenience here and further-on). We can clearly see by inspection of $\mathbf{M}_{\mathrm{coh}}$ how the direct coupling transfers coherence between the two lower transitions $l_{i}$, on the one hand, and the two upper transitions $u_{i}$, on the other hand. In this case, coherence can follow a closed path forming loops that flow always at the same rate $2 g$ (in between $u_{1} \leftrightarrow u_{2}$ and $d_{1} \leftrightarrow d_{2}$ ) and give rise to Rabi oscillations and dressed states.

In the case of a $4 \mathrm{LS}$, the situation is not much different, even when adding pump and decay: dressed states are also identified through the same procedure, diagonalizing the corresponding matrix without incoherent crossed terms:

$$
\mathbf{M}_{\mathrm{coh}}=\left(\begin{array}{cccc}
\frac{\Gamma_{1}}{2}+\gamma_{2} & 0 & -i g & 0 \\
0 & \frac{\Gamma_{1}}{2}+P_{2} & 0 & i g \\
-i g & 0 & \frac{\Gamma_{2}}{2}+\gamma_{1}-i \Delta & 0 \\
0 & i g & 0 & \frac{\Gamma_{2}}{2}+P_{1}-i \Delta
\end{array}\right) .
$$

In this case, the eigenenergies we found in Eq. 31) correspond to the same combination of transitions, Eqs. 58 59] with:

$$
| \pm\rangle \propto \frac{\tilde{\Gamma}_{-} \pm i \tilde{R}}{g}|1,0\rangle+i|0,1\rangle .
$$

If $\tilde{\Gamma}_{-}=0(\tilde{R}=g)$, we recover the dressed states without decoherence. Up to the transition into WC at $\left|\tilde{\Gamma}_{-}\right|=g$, both coefficients in Eq. (62) are complex numbers with unit norm and, therefore, they give rise to a balanced contribution of the two states $|1,0\rangle$ and $|0,1\rangle$ equal to $1 / 2$. Their mean energy, $\langle \pm|H| \pm\rangle= \pm \tilde{R}$, is exactly the one appearing in the spectral resonances $\omega_{p}$. The straightforward identification of dressed states, in the same way as when there is only direct coupling, makes it possible to describe the dynamics in the manifold picture as explained in appendix $D$

When the system is in WC $(\tilde{R}=i|\tilde{R}|)$, the dressed states become essentially the bare ones, $|1,0\rangle$ and $|0,1\rangle$, since $\left|\tilde{\Gamma}_{-}\right| / g \rightarrow \infty$. That is, in this regime, the populations become less and less balanced, as we can see in the example of Fig.12, where the probability to find the system in $|1,0\rangle$ is plotted in thin dashed for both $| \pm\rangle$.

On the other hand, in the case of two qubits which are only pumped and decaying, with no direct coupling, the eigenstates of $\mathbf{M}_{\mathrm{coh}}$ are combinations of the transitions corresponding to each qubit: $\left\langle P_{2} u_{1}+\gamma_{2} d_{1}\right\rangle,\left\langle P_{1} u_{2}+\gamma_{1} d_{2}\right\rangle,\left\langle u_{1}-d_{1}\right\rangle$ and $\left\langle u_{2}-\right.$ $\left.d_{2}\right\rangle$. No new dressed states arise although coherence is being transferred (incoherently) between two transitions.

From these three limiting situations, we learn that direct coupling is essential for the appearance of dressed states but also that pump and decay can transfer coherence in the directions $u_{1} \leftrightarrow d_{1}$ and $u_{2} \leftrightarrow d_{2}$, in an incoherent continuous way. In spite of this, they do not induce oscillations in the dynamics. Even in the most symmetrical case where $\gamma_{1}=P_{1}$ and $\gamma_{2}=P_{2}$, pump and decay rather move around coherence in an

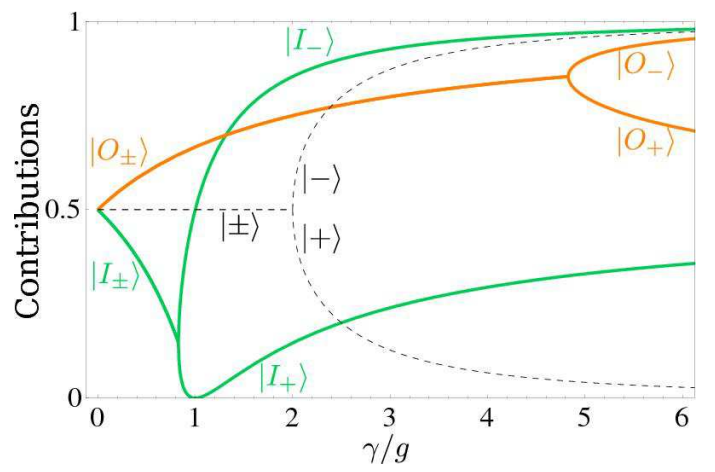

FIG. 12: (Color online) (a) Contributions from state $|1,0\rangle$ to the dressed states appearing in an optimally pumped case: $P_{1}=\gamma_{2}=\gamma$, $P_{2}=\gamma_{1}=0$. The $x$-axis is $\gamma / g$, as in previous Figs. 8 and 9 describing the same case. In thin dashed black lines, the contribution to the 4LS dressed states $| \pm\rangle$. In thick lines, the qubit dressed states: orange for $\left|O_{ \pm}\right\rangle$and green for $\left|I_{ \pm}\right\rangle$. The splitting of each pair of lines \pm from equal to different contributions, marks their transition into WC. All the "-" states tend towards $|1,0\rangle$ in the very weakly coupled regime.

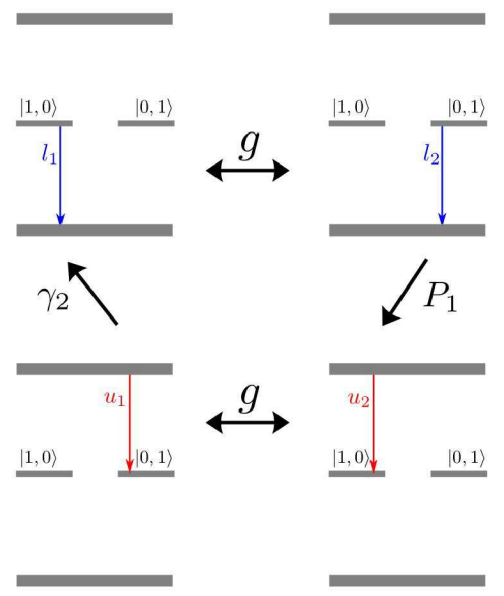

FIG. 13: (Color online) Circulation of coherence among the transitions between bare states for two qubits in the case where $\gamma_{1}=P_{2}=0$. A loop is formed that involves all the transitions, thanks to the pumping and decay terms that participate to the flow of coherence in a constructive way. The direct coupling goes both in tune with the loop or against it. When $\gamma_{2}=P_{1}=2 g$ the transfer is optimal.

incoherent way, basically disrupting the Rabi oscillations, as we observed at the end of Sec. $\mathrm{VIB}, G \leq g$.

Exceptionally, if the coherence transfer induced by pump and decay happens in a constructive, rather than independent, way as compared to the one induced by the direct coupling, Rabi oscillations and dressed states can be enhanced. This is the case of the optimally pumped configurations studied in Sec. VIA Let us analyze, for instance, the best situation, $\gamma_{2}=P_{1}=0$ and $\gamma_{1}=P_{2}=\gamma$, where $G=\sqrt{2} g$. In this case, the coherence transfer in the system can take place in a loop that involves not only the direct coupling but also incoherent processes, as sketched in Fig. 13 This results in the appearance of four different resonances in the system, that can be written 
in terms of two pairs of new intermediate dressed states,

$$
\begin{aligned}
& \left|I_{ \pm}\right\rangle \propto \frac{\gamma / 2 \pm i z_{1}}{g}|1,0\rangle+i|0,1\rangle, \\
& \left|O_{ \pm}\right\rangle \propto \frac{\gamma / 2 \pm i z_{2}}{g}|1,0\rangle+i|0,1\rangle,
\end{aligned}
$$

named after inner and outer eigenenergies. They correspond to $\gamma \pm i z_{1}$ and $\gamma \pm i z_{2}$, respectively, where $z_{1,2}$ are those in Eq. (47). The level scheme that these new coherence loops produce is plotted in Fig. 22 d). In SSC, the dressed states appear in the eigentransitions as:

$$
\begin{aligned}
& \left\langle\mathrm{T}_{A}\right\rangle=\left\langle\mid I_{-}\right\rangle\langle 1,1|+i| 0,0\rangle\left\langle I_{+} \mid\right\rangle, \\
& \left\langle\mathrm{T}_{B}\right\rangle=\left\langle\mid O_{-}\right\rangle\langle 1,1|-i| 0,0\rangle\left\langle O_{+} \mid\right\rangle, \\
& \left\langle\mathrm{T}_{C}\right\rangle=\left\langle\mid I_{+}\right\rangle\langle 1,1|+i| 0,0\rangle\left\langle I_{-} \mid\right\rangle, \\
& \left\langle\mathrm{T}_{D}\right\rangle=\left\langle\mid O_{+}\right\rangle\langle 1,1|-i| 0,0\rangle\left\langle O_{-} \mid\right\rangle .
\end{aligned}
$$

The normalization of these dressed states also depends on the coupling region. In SSC, inner states are normalized by dividing by $\sqrt{g(2 g-\gamma)}$ and outer by $\sqrt{g(2 g+\gamma)}$, instead of $\sqrt{2} g$ as was the case of the 4LS. These states do not have a balanced contribution from the bare states due to the saturation brought by pump and decay. The inner states have a smaller contribution from the first qubit than the outer states:

$$
\begin{aligned}
& \left|\left\langle 1,0 \mid I_{ \pm}\right\rangle\right|^{2}=\frac{1}{2}\left(1-\frac{\gamma}{2 g-\gamma}\right), \\
& \left|\left\langle 1,0 \mid O_{ \pm}\right\rangle\right|^{2}=\frac{1}{2}\left(1+\frac{\gamma}{2 g+\gamma}\right) .
\end{aligned}
$$

The 4LS dressed states correspond to an intermediate situation between the inner and outer dressed states in terms of populations (equal to $1 / 2$ for both qubit) and energy (see Fig. 10. This appears clearly in Fig. 12 where the first qubit contribution to each dressed state is plotted as a function of $\gamma / \mathrm{g}$.

The two transitions composing each of the eigentransitions in Eq. (63) have the same energy, which does not coincide with the average Hamiltonian value as soon as decoherence appears: $\left\langle I_{ \pm}|H| I_{ \pm}\right\rangle= \pm \Re\left(z_{1}\right) /[1-\gamma /(2 g)]$ and $\left\langle O_{ \pm}|H|\right.$ $\left.O_{ \pm}\right\rangle= \pm \Re\left(z_{2}\right) /[1+\gamma /(2 g)]$.

When entering WC, the splittings between each pair of dressed states close, while the eigentransitions are linked each to a single state:

$$
\begin{aligned}
& \left\langle\mathrm{T}_{A}\right\rangle=\left\langle\mid I_{-}\right\rangle\langle 1,1|+i| 0,0\rangle\left\langle I_{-} \mid\right\rangle, \\
& \left\langle\mathrm{T}_{B}\right\rangle=\left\langle\mid O_{-}\right\rangle\langle 1,1|+i| 0,0\rangle\left\langle O_{-} \mid\right\rangle, \\
& \left\langle\mathrm{T}_{C}\right\rangle=\left\langle\mid I_{+}\right\rangle\langle 1,1|+i| 0,0\rangle\left\langle I_{+} \mid\right\rangle, \\
& \left\langle\mathrm{T}_{D}\right\rangle=\left\langle\mid O_{+}\right\rangle\langle 1,1|+i| 0,0\rangle\left\langle O_{+} \mid\right\rangle .
\end{aligned}
$$

Note that the inner peaks are always the most intense ones and that, when entering the WC, it is the "-" states that dominate the spectrum due to the saturation of the system into their limiting state $|1,0\rangle$.

\section{CONCLUSIONS}

I described fully analytically the weak and strong coupling of two qubits in presence of dissipation and pumping. This configuration is realized by two two-level systems that commute. This was contrasted with other models of quantum coupling between two modes, namely, two bosons (that commute) and two fermions (that anticommute). I also addressed the case of a four-level system (4LS), which has the same level structure.

Whereas the coupling of bosons, fermions and a 4LS manifest a conventional phenomenology, well known from the normal coupling of two modes and differing only in bosonic or fermionic effective parameters, I showed that cross-Lindblad terms in the two coupled qubits give rise to a rich and complex new nomenclature of coupling. Namely, beyond standard strong coupling (SC) where two dressed states emerge from quantum superposition of the bare states, I found that four dressed states, $\left|I_{ \pm}\right\rangle$and $\left|O_{ \pm}\right\rangle$, can be realized in the system with two different Rabi splittings, as a result of pumping and decay establishing new paths of coherence flow in the system. This is a new paradigm of strong coupling that leads to new regimes that I called "Second order Strong Coupling" (SSC), when the four dressed states are split (in two pairs), and "Mixed Coupling" (MC), when one pair of dressed states, the inner $\left|I_{ \pm}\right\rangle$, have closed. In this wider picture, I called "First order Strong Coupling" (FSC) the conventional type of strong coupling with only one pair of dressed states. These results show and spell out the considerable complexity of coherence transfer in other systems such as the Jaynes-Cumming model in presence of incoherent pumping, that are not amenable to analytical treatment.

\section{Acknowledgments}

I thank F. P. Laussy for fruitful discussions and critical reading of the manuscript and T. Ostatnický for useful comments. This research was supported by the Newton Fellowship program.

\section{Appendix A: Interactions and dephasing}

If the two qubits are close enough in space, they may interact. The energy levels are affected by the interaction (Coulomb interaction in the case of atoms or excitons) and the Hamiltonian must be corrected with the term:

$$
H_{\mathrm{b}}=-\chi \sigma_{1}^{\dagger} \sigma_{1} \sigma_{2}^{\dagger} \sigma_{2},
$$

where $\chi$ is the binding energy between two excitations $(\chi>0$ corresponds to attraction). If $\chi \ll \omega_{1,2}$, interactions have a negligible effect on the populations and statistics, but not on the spectral features, starting with Eq. (4b) becoming $\omega_{11}=$ $\omega_{1}+\omega_{2}-\chi$. The dynamics in the presence of interactions is derived in appendix $B$ although the expressions for spectrum and dressed states are not presented in the text as they are not analytical in the most general case, and bring little to the discussion. The main effect that it produces is that upper and lower transitions can be identified thanks to the different energy of the upper transitions and this was used to check the 
consistency of the results. In FSC and WC, the difference is already present due to the broadenings associated to each transition and interactions only accentuate it. However, in SSC and $\mathrm{MC}$, interactions break the symmetry between upper and lower transitions (e.g., $\omega_{A}$ was linked to both upper or lower transition from inner states in Fig. 2(d)) depending on the sign of $\chi$ :

- if $\chi>0, \omega_{A, D}$ correspond to the upper transitions and $\omega_{B, C}$ to the lower transitions

- if $\chi<0, \omega_{A, D}$ correspond to the lower transitions and $\omega_{B, C}$ to the upper transitions

Naturally, strong interactions produce splittings between the peaks corresponding to upper and lower transitions, which may turn the spectral doublets into fully formed quadruplets.

A second element that may be important in the system is pure dephasing. It may be caused, depending on the physical system, by interaction with phonons or other external elements. This effect has proved to be relevant in state of the art semiconductor experiments [38]. Noting the rates of dephasing of each qubit as $\delta_{1}, \delta_{2}$, one must solve the following extended master equation from Eq. 5):

$$
\frac{d \rho}{d t}=\mathscr{L} \rho+\sum_{i=1,2} \frac{\delta_{i}}{2}\left(2 \sigma_{i}^{\dagger} \sigma_{i} \rho \sigma_{i}^{\dagger} \sigma_{i}-\sigma_{i}^{\dagger} \sigma_{i} \rho-\rho \sigma_{i}^{\dagger} \sigma_{i}\right)
$$

In the formalism, as a consequence, Eq. (7a) becomes:

$$
\Gamma_{1}^{d}=\gamma_{1}+P_{1}+\delta_{1}, \quad \Gamma_{2}^{d}=\gamma_{2}+P_{2}+\delta_{2}
$$

The dynamics in the presence of pure dephasing is again derived in appendix $B$ and not discussed in the main text for the same reason as before, that it spoils analyticity of the results and brings little to the discussion. The main effect of pure dephasing is to weaken the coherent coupling and is of a quantitative nature, not relevant enough to discuss it further in the present text.

\section{Appendix B: Quantum regression formula and time correlators}

In this appendix I obtain, thanks to the quantum regression formula, the first order differential equations for the one and two-time correlators required for the spectrum in Eq. 10. The quantum regression theorem states that, once we find the set of operators $C_{\{\eta\}}$ and the regression matrix $M$ that sat-

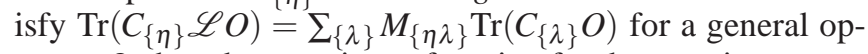
erator $O$, then the equations of motion for the two-time correlators $(\tau \geq 0)$ read:

$$
\frac{\partial}{\partial \tau}\left\langle O(t) C_{\{\eta\}}(t+\tau)\right\rangle=\sum_{\{\lambda\}} M_{\{\eta \lambda\}}\left\langle O(t) C_{\{\lambda\}}(t+\tau)\right\rangle
$$

The most general set of operators for the two coupled qubits is $C_{\{m, n, \mu, v\}}=\sigma_{1}^{\dagger m} \sigma_{1}^{n} \sigma_{2}^{\dagger \mu} \sigma_{2}^{v}$, with $m, n, \mu, v \in\{0,1\}$. The corresponding regression matrix $M$ is:

$$
\begin{aligned}
& M_{m n \mu v}=i \omega_{1}(m-n)+i \omega_{2}(\mu-v)-i \chi(m \mu-n v) \\
& -\frac{\Gamma_{1}}{2}(m+n)-\frac{\Gamma_{2}}{2}(\mu+v)-\frac{\delta_{1}}{2}(m-n)^{2}-\frac{\delta_{2}}{2}(\mu-v)^{2}, \\
& M_{1-m, 1-n, \mu v}=P_{1} m n-i \chi(1-m)(1-n)(\mu-v), \\
& M_{m n, 1-\mu, 1-v}{ }_{m i v}
\end{aligned}
$$

and zero everywhere else.

We concentrate on computing $G_{1}^{(1)}$, as $G_{2}^{(1)}$ can be obtained from it by exchanging the indexes $1 \leftrightarrow 2$. It corresponds to setting $O=\sigma_{1}^{\dagger}$ and having $\{m, n, \mu, v\}=\{0,1,0,0\}$ in Eq. B1]. Other two-time correlators are linked to the one of interest by the regression matrix, and therefore are also needed to compute the spectrum. All correlators can be grouped in manifolds that, following Refs [14, 16, 36], I denote $\mathscr{N}_{k}$, where $k$ is the minimum number of particles that allows the correlators to take a nonzero value. As we can see schematically in the left part of Fig. 14 the first manifold $\mathscr{N}_{1}$ is composed of the correlators with $\{0,1,0,0\}$ and $\{0,0,0,1\}$, linked only by the coherent coupling (red arrows). In the linear regime (very low pump), it is enough to solve the equations truncating in this manifold, which is the same as in the linear model and includes $G_{1}^{(1)}(\tau)$. The next (and last) manifold $\mathscr{N}_{2}$, where the two models differ, is composed of the correlators with $\{1,1,0,1\}$ and $\{0,1,1,1\}$. The links between manifolds are also of an incoherent nature, due to the pump (green and blue arrows).

Gathering the four correlators in a vector

$$
\mathbf{v}(t, t+\tau)=\left(\begin{array}{c}
\left\langle\sigma_{1}^{\dagger}(t) \sigma_{1}(t+\tau)\right\rangle \\
\left\langle\sigma_{1}^{\dagger}(t) \sigma_{2}(t+\tau)\right\rangle \\
\left\langle\sigma_{1}^{\dagger}(t) \sigma_{1}^{\dagger} \sigma_{1} \sigma_{2}(t+\tau)\right\rangle \\
\left\langle\sigma_{1}^{\dagger}(t) \sigma_{1} \sigma_{2}^{\dagger} \sigma_{2}(t+\tau)\right\rangle
\end{array}\right)
$$

their equations of motion read in matricial form:

$$
\frac{\partial}{\partial \tau} \mathbf{v}(t, t+\tau)=-\mathbf{M}_{1} \mathbf{v}(t, t+\tau)
$$

with

$\mathbf{M}_{1}=$

$\left(\begin{array}{cccc}i \omega_{1}+\frac{\Gamma_{1}^{\mathrm{d}}}{2} & i g & -2 i g & -i \chi \\ i g & i \omega_{2}+\frac{\Gamma_{2}^{\mathrm{d}}}{2} & -i \chi & -2 i g \\ 0 & -P_{1} & i\left(\omega_{2}-\chi\right)+\frac{2 \Gamma_{1}+\Gamma_{2}^{\mathrm{d}}}{2} & -i g \\ -P_{2} & 0 & -i g & i\left(\omega_{1}-\chi\right)+\frac{2 \Gamma_{2}+\Gamma_{1}^{\mathrm{d}}}{2}\end{array}\right)$ 


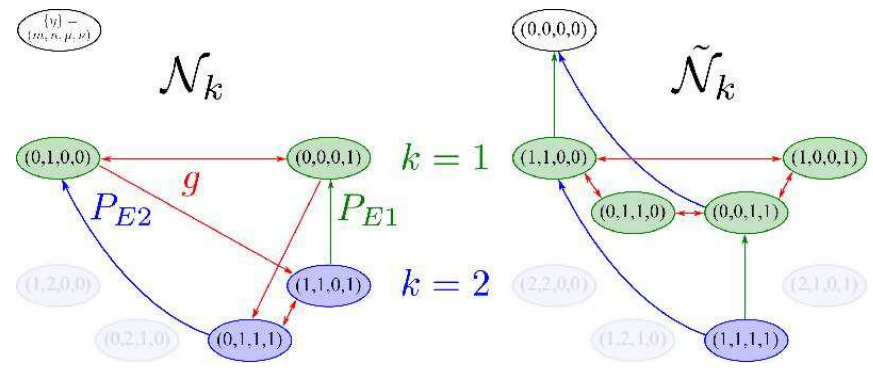

FIG. 14: (Color online) Chain of correlators-indexed by $\{\eta\}=$ $(m, n, \mu, v)$-linked by the Hamiltonian dynamics with pump and decay for two coupled qubits. On the left (resp., right), the set $\bigcup_{k} \mathscr{N}_{k}$ (resp., $\bigcup_{k} \tilde{\mathscr{N}}_{k}$ ) involved in the equations of the two-time (resp., single-time) correlators. In green are shown the first manifolds $\mathscr{N}_{1}$ and $\tilde{\mathscr{N}}_{1}$ that correspond to the linear model [14], and in blue, the second manifold $\mathscr{N}_{2}$ and $\tilde{\mathscr{N}}_{2}$. The equation of motion $\left\langle\sigma_{1}^{\dagger}(t) C_{\{\eta\}}(t+\tau)\right\rangle$ with $\eta \in \mathscr{N}_{k}$ requires for its initial value the correlator $\left\langle C_{\{\tilde{\eta}\}}\right\rangle$ with $\{\tilde{\eta}\} \in \tilde{\mathscr{N}}_{k}$ defined from $\{\eta\}=(m, n, \mu, v)$ by $\{\tilde{\eta}\}=(m+1, n, \mu, v)$, as seen on the diagram. The thick red arrows indicate which elements are linked by the coherent (SC) dynamics, through the coupling strength $g$, while the green/blue thin arrows show the connections due to the incoherent quantum dot pumpings. The sense of the arrows indicates which element is "calling" which in its equations. The self-coupling of each node to itself is not shown. This is where $\omega_{1,2}$ and $\Gamma_{1,2}$ enter. These links are obtained from the rules in Eqs. B2], that result in the matrices $\mathbf{M}_{1}$ and $\mathbf{M}_{0}$. The number of correlators needed to compute the spectrum is truncated naturally (with four elements) thanks to the saturation of the qubit.

The general solution (for $\tau \geq 0$ ), $\mathbf{v}(t, t+\tau)=e^{-\mathbf{M}_{1} \tau} \mathbf{v}(t, t)$, leads to the correlator in Eq. (12) with only four contributions, indexed by $p=A, B, C$ and $D$. The frequencies $\omega_{p}$ and rates $\gamma_{p}$ are the imaginary and real parts of the eigenvalues of the matrix $\mathbf{M}_{1}$. The dimensionless coefficients $L_{p}(t)$ and $K_{p}(t)$, also real, are linear combinations of the single-time average quantities,

$$
\mathbf{v}(t, t)=\left(\begin{array}{c}
\left\langle\sigma_{1}^{\dagger} \sigma_{1}\right\rangle(t) \\
\left\langle\sigma_{1}^{\dagger} \sigma_{2}\right\rangle(t) \\
\left\langle\sigma_{1}^{\dagger} \sigma_{1}^{\dagger} \sigma_{1} \sigma_{2}\right\rangle(t) \\
\left\langle\sigma_{1}^{\dagger} \sigma_{1} \sigma_{2}^{\dagger} \sigma_{2}\right\rangle(t)
\end{array}\right) .
$$

These mean values can also be found through the quantum regression formula, setting the operator $O=1$ and deriving the corresponding regression matrix from the rules in Eq. (B2). They can also be grouped in manifolds, denoted $\mathscr{N}_{k}$. All nonzero single-time average quantities, all of them needed to compute the spectrum, are shown in the right part of Fig. 14. Note that $\left\langle\sigma_{1}^{\dagger} \sigma_{1}^{\dagger} \sigma_{1} \sigma_{2}\right\rangle(t)=0$. The correlators in $\tilde{\mathscr{N}}_{1}$ can be obtained independently solving a second matricial equation:

$$
\frac{d \mathbf{u}(t)}{d t}=-\mathbf{M}_{0} \mathbf{u}(t)+\mathbf{p}
$$

with

$$
\begin{gathered}
\mathbf{u}(t)=\left(\begin{array}{c}
\left\langle\sigma_{1}^{\dagger} \sigma_{1}\right\rangle(t) \\
\left\langle\sigma_{2}^{\dagger} \sigma_{2}\right\rangle(t) \\
\left\langle\sigma_{1}^{\dagger} \sigma_{2}\right\rangle(t) \\
\left\langle\sigma_{2}^{\dagger} \sigma_{1}\right\rangle(t)
\end{array}\right), \quad \mathbf{p}=\left(\begin{array}{c}
P_{1} \\
P_{2} \\
0 \\
0
\end{array}\right), \\
\mathbf{M}_{0}=\left(\begin{array}{cccc}
\Gamma_{1} & 0 & i g & -i g \\
0 & \Gamma_{2} & -i g & i g \\
i g & -i g & \frac{\Gamma_{1}^{\mathrm{d}}+\Gamma_{2}^{\mathrm{d}}}{2}-i \Delta & 0 \\
-i g & i g & 0 & \frac{\Gamma_{1}^{\mathrm{d}}+\Gamma_{2}^{\mathrm{d}}}{2}+i \Delta
\end{array}\right) .
\end{gathered}
$$

This equation is similar to that of the linear model, only changing the effective fermionic rates into bosonic ones $\Gamma_{i} \rightarrow$ $\tilde{\Gamma}_{i}$ [14]. On the other hand, the correlator with $\{1,1,1,1\}$ (that is, $\left\langle\sigma_{1}^{\dagger} \sigma_{1} \sigma_{2}^{\dagger} \sigma_{2}\right\rangle(t)$ ), which is the only one that is not zero in $\tilde{N}_{2}$, finds its expression separately, only in terms of the operators labeled $\{1,1,0,0\}$ and $\{0,0,1,1\}$, through the equation

$$
\begin{aligned}
\frac{d}{d t}\left\langle\sigma_{1}^{\dagger} \sigma_{1} \sigma_{2}^{\dagger} \sigma_{2}\right\rangle(t)=-4 \Gamma_{+}\left\langle\sigma_{1}^{\dagger} \sigma_{1} \sigma_{2}^{\dagger} \sigma_{2}\right\rangle(t) \\
+P_{2}\left\langle\sigma_{1}^{\dagger} \sigma_{1}\right\rangle(t)+P_{1}\left\langle\sigma_{2}^{\dagger} \sigma_{2}\right\rangle(t)
\end{aligned}
$$

In order to insert these averages in the expression (10) for the spectrum, they must be either time integrated, in the spontaneous emission case (SE), to give $\mathbf{v}^{\mathrm{SE}}=\int_{0}^{\infty} \mathbf{v}(t, t) d t$ (and the coefficients $L_{p}^{\mathrm{SE}}$ and $K_{p}^{\mathrm{SE}}$ ) or computed directly in the steady state (SS) to give $\mathbf{v}^{\mathrm{SS}}=\lim _{t \rightarrow \infty} \mathbf{v}(t, t)$ (and the coefficients $\left.L_{p}^{\mathrm{SS}}, K_{p}^{\mathrm{SS}}\right)$.

\section{Two fermions}

The regression matrix $\mathbf{M}_{1}$ (cf Eq. B5) truncates to its first submatrix,

$$
\mathbf{M}_{1}=\left(\begin{array}{cc}
i \omega_{1}+\frac{\Gamma_{1}^{d}}{2} & i g \\
i g & i \omega_{2}+\frac{\Gamma_{2}^{d}}{2}
\end{array}\right) .
$$

recovering the exact mapping to the linear model, as discussed in the text.

\section{Matrix of coherences}

The matrix of coherence in Eq. 54 can be found by rewriting the regression matrix $\mathbf{M}_{1}$ in the basis of projectors for each transition, presented in Eq. [53): $\mathbf{M}_{\mathrm{coh}}=\mathbf{A}^{-1} \mathbf{M}_{1} \mathbf{A}$ with A some transformation matrix. For this reason, they have the same eigenvalues, which are the resonances of the systems. Since the eigenstates of $\mathbf{M}_{\mathrm{coh}}$ are the transitions between dressed states, their study enables us to reconstruct them even in a highly dissipative environment. 


\section{Appendix C: Cross Lindblad terms}

In the case where the four levels of the system do not correspond to two qubits but are the levels of a single entity [32], the corresponding Liouvillian of the system is most conveniently written in terms of the four-level operators in Eq. (6). The final master equation in that case can be found from $\mathscr{L} \rho$ in Eq. (5) by removing cross terms that appear in the two coupled qubits:

$$
\begin{aligned}
\frac{d \rho}{d t}=\mathscr{L} \rho & -\gamma_{1}\left(l_{1} \rho u_{1}^{\dagger}+u_{1} \rho l_{1}^{\dagger}\right)-\gamma_{2}\left(l_{2} \rho u_{2}^{\dagger}+u_{2} \rho l_{2}^{\dagger}\right) \\
& -P_{1}\left(u_{1}^{\dagger} \rho l_{1}+l_{1}^{\dagger} \rho u_{1}\right)-P_{2}\left(u_{2}^{\dagger} \rho l_{2}+l_{2}^{\dagger} \rho u_{2}\right) \\
& -\delta_{1}\left(l_{1}^{\dagger} l_{1} \rho u_{1}^{\dagger} u_{1}+u_{1}^{\dagger} u_{1} \rho l_{1}^{\dagger} l_{1}\right) \\
& -\delta_{2}\left(l_{2}^{\dagger} l_{2} \rho u_{2}^{\dagger} u_{2}+u_{2}^{\dagger} u_{2} \rho l_{2}^{\dagger} l_{2}\right) .
\end{aligned}
$$

Such cross terms represent effective couplings between the coherences, in the case of pump and decay, and the states, in the case of pure dephasing, associated to each qubit. Evidently, they can only have consequences on the dynamics when the two qubits are also directly coupled. The rules to add to those in Eq. (B2) in order to obtain a new general regression matrix $M+\delta M$ are:

$\underset{m \text { mn } \mu v}{\delta M_{\text {miv }}}=P_{1}(m+n-1)(\mu-v)^{2}+P_{2}(\mu+v-1)(m-m)^{2}$,

$\delta M \underset{1-m, 1-n, \mu v}{m n \mu v}=-(\mu-v)^{2}\left[\left(P_{1}+\delta_{1}\right) m n-\tilde{\Gamma}_{1}(1-m)(1-n)\right]$,

$\delta M_{m n, 1-\mu, 1-v}^{m n \mu v}=-(m-n)^{2}\left[\left(P_{2}+\delta_{2}\right) \mu v-\tilde{\Gamma}_{2}(1-\mu)(1-v)\right]$.

With these new rules, the matrix for the one-time correlators, $\mathbf{M}_{0}$, is still the same as in Eq. (B8b) and, therefore, the mean values in $\mathbf{u}(t)$, Eq. (B8a), do not differ from those for two qubits. The two-time regression matrix $\mathbf{M}_{1}$ changes into $\mathbf{M}_{1}+$ $\delta \mathbf{M}_{1}$ with

$$
\delta \mathbf{M}_{1}=\left(\begin{array}{cccc}
P_{2} & 0 & 0 & \gamma_{2}-P_{2} \\
0 & P_{1} & \gamma_{1}-P_{1} & 0 \\
0 & \delta_{1}+P_{1} & -P_{1} & 0 \\
\delta_{2}+P_{2} & 0 & 0 & -P_{2}
\end{array}\right) .
$$

\section{Appendix D: Dressed states in the manifold picture}

We can give an alternative derivation and intuitive interpretation of the results in section $\mathrm{VA}$ by assuming complex frequencies $\omega_{1,2} \rightarrow \Omega_{1,2}=\omega_{1,2}-i \gamma_{1,2} / 2$ in Eq. (3). This leads to the complex dressed frequencies:

$$
\begin{aligned}
& \omega_{+} \rightarrow \Omega_{+}=\omega_{1}-i \gamma_{+} \pm R_{0}, \\
& \omega_{11} \rightarrow \Omega_{11}=2 \omega_{1}-2 i \gamma_{+} .
\end{aligned}
$$

From here, we can compute positions and broadenings of the four possible contributions to the spectra. The positions are given by the difference in energy between the levels involved in the transitions while the broadenings are given by the sum of the imaginary parts (the uncertainties). If we apply this principle between the levels in Eq. (D1), we find the same result than in Eq. (34)-(35). This allows us to identify the contributions $A, B, C, D$ to the $\mathrm{SC}$ spectrum with the manifold transitions in the order that we now explain. In Fig. 2 (b) we can follow the four possible transitions in the manifold picture. The lower transitions (in blue), from $| \pm\rangle$, coincide, respectively, with the expressions for peaks $A$ and $D$, given by Eq. (34). The upper transitions (in red), towards $| \pm\rangle$, coincide with the expressions for peaks $B$ and $C$, given by Eq. (34). The upper transitions have a larger broadening than the lower due to the addition of the uncertainties in energy of the levels involved, brought by the spontaneous decay.

In order to understand better some features of the spectra of the steady state under incoherent pump in the different regions that we have defined in section $\mathrm{VB}$, we will now attempt to push the manifold method - adequate for vanishing pump-a bit further, to the case of non-negligible pump. If we combine their effects in the non-hermitian Hamiltonian as

$$
\begin{aligned}
& \Omega_{0,0}=-i \frac{P_{1}+P_{2}}{2}, \\
& \Omega_{ \pm}=\omega_{1}-i \Gamma_{+} \pm R^{1 \mathrm{TD}}, \\
& \Omega_{11}=2 \omega_{1}-i \frac{\gamma_{1}+\gamma_{2}}{2},
\end{aligned}
$$

and apply their sum and subtraction to obtain positions and broadenings of the spectral contributions of each of the four transitions:

$$
\begin{gathered}
\frac{\gamma_{A}}{2}+i \omega_{A}=\frac{3\left(P_{1}+P_{2}\right)+\gamma_{1}+\gamma_{2}}{4}+i R^{1 \mathrm{TD}}, \\
\frac{\gamma_{B}}{2}+i \omega_{B}=\frac{3\left(\gamma_{1}+\gamma_{2}\right)+P_{1}+P_{2}}{4}+i R^{1 \mathrm{TD}}, \\
\frac{\gamma_{C}}{2}+i \omega_{C}=\frac{3\left(\gamma_{1}+\gamma_{2}\right)+P_{1}+P_{2}}{4}-i R^{1 \mathrm{TD}}, \\
\frac{\gamma_{D}}{2}+i \omega_{D}=\frac{3\left(P_{1}+P_{2}\right)+\gamma_{1}+\gamma_{2}}{4}-i R^{1 \mathrm{TD}},
\end{gathered}
$$

we do not obtain the right results of four-in principle different—peaks. This discussion brings us back to the naive association of SC with $\Re\left(R^{1 \mathrm{TD}}\right) \neq 0$ we discarded in Sec. III. It is evident that using only the single-time dynamics frequency $R^{1 \mathrm{TD}}$ to describe the splitting between the dressed states, cannot give the variety of situations than the two complex parameters $z_{1,2}$ give.

In the case of a $4 \mathrm{LS}$, the results obtained in Eq. 31] are comparable to those in Eq. (D3), even though the positions of the peaks are given by $\tilde{R}$ instead of $R^{1 \mathrm{TD}}$. The broadenings are exactly the same. We can conclude that without the cross Lindblad terms, the coupling acquires a bosonic character: the correlations between levels are relaxed from those of two qubits into those of one (larger) four-level system where the manifold picture holds. 


\section{Appendix E: Second order correlations functions}

In this appendix, I obtain the second order direct and cross correlation functions defined, respectively, as

$$
\begin{aligned}
& G_{1}^{(2)}(t, t+\tau)=\left\langle\sigma_{1}^{\dagger}(t) \sigma_{1}^{\dagger}(t+\tau) \sigma_{1}(t+\tau) \sigma_{1}(t)\right\rangle, \\
& G_{12}^{(2)}(t, t+\tau)=\left\langle\sigma_{1}^{\dagger}(t) \sigma_{2}^{\dagger}(t+\tau) \sigma_{2}(t+\tau) \sigma_{1}(t)\right\rangle .
\end{aligned}
$$

These two quantities are proportional to the probabilities of two $\tau$-delayed emissions from the same qubit $\left(G_{1}^{(2)}\right)$ or two different qubit emissions $\left(G_{12}^{(2)}\right)$.

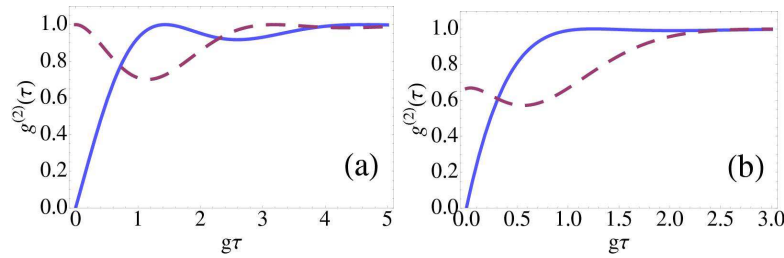

FIG. 15: (Color online) Steady state values of $g^{(2)}(\tau)$ (solid blue) $g_{12}^{(2)}(\tau)$ (dashed purple) at resonance. (a) The system is in FSC: $\gamma_{1}=$ $g, \gamma_{2}=g / 2, P_{1}=g / 2$ and $P_{2}=g / 10$. (b) In MC with optimum coupling $G=\sqrt{2} g: \gamma_{1}=P_{2}=0$ and $P_{1}=\gamma_{2}=2 g$ (see Fig. 8).

In order to compute such two-time correlators, we apply the quantum regression formula presented in an alternative form to Eq. (B1):

$$
\frac{\partial}{\partial \tau}\left\langle O(t) C_{\{\eta\}}(t+\tau) Q(t)\right\rangle=\sum_{\{\lambda\}} M_{\{\eta \lambda\}}\left\langle O(t) C_{\{\lambda\}}(t+\tau) Q(t)\right\rangle
$$

where $Q$ is a second arbitrary operator. The equations we must solve setting $O=\sigma_{1}^{\dagger}$ and $Q=\sigma_{1}$, are the same as those used to compute the mean values in Eq. B7 but now with the vector of correlators

$$
\mathbf{w}(t, t+\tau)=\left(\begin{array}{c}
\left\langle\sigma_{1}^{\dagger}(t) \sigma_{1}^{\dagger} \sigma_{1}(t+\tau) \sigma_{1}(t)\right\rangle \\
\left\langle\sigma_{1}^{\dagger}(t) \sigma_{2}^{\dagger} \sigma_{2}(t+\tau) \sigma_{1}(t)\right\rangle \\
\left\langle\sigma_{1}^{\dagger}(t) \sigma_{1}^{\dagger} \sigma_{2}(t+\tau) \sigma_{1}(t)\right\rangle \\
\left\langle\sigma_{1}^{\dagger}(t) \sigma_{1} \sigma_{2}^{\dagger}(t+\tau) \sigma_{1}(t)\right\rangle
\end{array}\right),
$$

and the vector $n_{1}(t) \mathbf{p}$ instead of $\mathbf{p}$. In the steady state $(t=0)$, the solution for $\mathbf{w}(0, \tau)$ requires knowing the initial condition:

$$
\mathbf{w}^{\mathrm{SS}}=\mathbf{w}(0,0)=\left(\begin{array}{c}
0 \\
n_{11} \\
0 \\
0
\end{array}\right)
$$

In terms of the following vector of mean fluctuations,

$$
\mathbf{f}^{\mathrm{SS}}=-\left(\mathbf{w}^{\mathrm{SS}}-\mathbf{u}^{\mathrm{SS}} n_{1}^{\mathrm{SS}}\right)=\left(\begin{array}{c}
n_{1}^{2} \\
n_{1} n_{2}-n_{11} \\
n_{\text {corr }} n_{1} \\
n_{\text {corr }}^{*} n_{1}
\end{array}\right),
$$

the steady state normalized correlations functions (for $\tau>0$ ) read

$$
\begin{aligned}
& g^{(2)}(\tau)=\frac{G_{1}^{(2)}(0, \tau)}{n_{1}^{2}}=1-\frac{\left[e^{-\mathbf{M}_{0} \tau} \mathbf{f}^{\mathrm{SS}}\right]_{1}}{n_{1}^{2}} \in[0,1], \\
& g_{12}^{(2)}(\tau)=\frac{G_{12}^{(2)}(0, \tau)}{n_{1} n_{2}}=1-\frac{\left[e^{-\mathbf{M}_{0} \tau} \mathbf{f}^{\mathrm{SS}}\right]_{2}}{n_{1} n_{2}} \in[0,1]
\end{aligned}
$$

where $[\mathbf{x}]_{1}$ means that we take the first element of the vector $\mathbf{x}$. At infinite delays, the two functions reach the value of uncorrelated emissions,

$$
g_{1}^{(2)}(\tau \rightarrow \infty), g_{12}^{(2)}(\tau \rightarrow \infty) \rightarrow 1
$$

At zero delay, it is evident that $g^{(2)}(0)=0$, as two excitations cannot exist in the same qubit. At intermediate delays, the emission presents antibunching: $g^{(2)}(0)<g^{(2)}(\tau)$, the second excitation from the same qubit has more probability to be emitted after some delay (see Fig. 15). At zero delay,

$$
g_{12}^{(2)}(0)=\frac{n_{11}}{n_{1} n_{2}}
$$

It always is possible a second emission coming from a different qubit (the minimum value for $g_{12}^{(2)}(\tau=0)$ is $\left.1 / 5\right)$. When the qubit behave as independently, $g_{12}^{(2)}(\tau)=1$ all along.

Being the second order correlation functions given by basically the same equation as the populations and depending only on them, $g_{1}^{(2)}(\tau)$ and $g_{12}^{(2)}(\tau)$ are the same for the two coupled fermions, qubits and 4LS. It is not possible to distinguish from their dynamics the four coupling regions described in Table appearing in the case of coupled qubits.
[1] M. A. Nielsen and I. L. Chuang, Quantum computation and quantum information (Cambridge University Press, 2000).

[2] G. W. Gardiner and P. Zoller, Quantum Noise (Springer-Verlag, Berlin, 2000), 2nd ed.

[3] S. Haroche and J.-M. Raimond, Exploring the Quantum: Atoms, Cavities, and Photons (Oxford University Press, 2006).
[4] L. Allen and J. H. Eberly, Optical Resonance and Two-Level Atoms (Dover, 1987).

[5] A. Kavokin, J. J. Baumberg, G. Malpuech, and F. P. Laussy, Microcavities (Oxford University Press, 2007).

[6] C. Cohen-Tannoudji, J. Dupont-Roc, and G. Grynberg, Photons et atomes (EDP Sciences, 2001). 
[7] J. J. Sanchez-Mondragon, N. B. Narozhny, and J. H. Eberly, Phys. Rev. Lett. 51, 550 (1983).

[8] H. J. Carmichael, R. J. Brecha, M. G. Raizen, H. J. Kimble, and P. R. Rice, Phys. Rev. A 40, 5516 (1989).

[9] B. R. Mollow, Phys. Rev. 188, 1969 (1969).

[10] C. Cohen-Tannoudji and S. Reynaud, J. phys. B.: At. Mol. Phys. 10, 345 (1977)

[11] J. I. Cirac, H. Ritsch, and P. Zoller, Phys. Rev. A 44, 4541 (1991).

[12] L. Tian and H. J. Carmichael, Quantum Opt. 4, 131 (1992).

[13] E. del Valle, Microcavity Quantum Electrodynamics (VDM Verlag, 2009).

[14] F. P. Laussy, E. del Valle, and C. Tejedor, Phys. Rev. B 79, 235325 (2009).

[15] E. Jaynes and F. Cummings, Proc. IEEE 51, 89 (1963).

[16] E. del Valle, F. P. Laussy, and C. Tejedor, Phys. Rev. B 79, 235326 (2009).

[17] Y. A. Pashkin, O. Astafiev, T. Yamamoto, Y. Nakamura, and J. S. Tsai, Quantum Information Processing 8, 55 (2009).

[18] Y. A. Pashkin, T. Yamamoto, O. Astafiev, Y. Nakamura, D. V. Averin, and J. S. Tsai, Nature 421, 823 (2003).

[19] J. B. Majer, F. G. Paauw, A. C. J. ter Haar, C. J. P. M. Harmans, and J. E. Mooij, Phys. Rev. Lett. 94, 090501 (2005).

[20] T. Hime, P. A. Reichardt, B. L. T. Plourde, T. L. Robertson, C.E. Wu, A. V. Ustinov, and J. Clarke, Science 314, 1427 (2006).

[21] A. O. Niskanen, K. Harrabi, F. Yoshihara, Y. Nakamura, S. Lloyd, and J. S. Tsai, Science 316, 723 (2007).

[22] W. K. Wootters, Phys. Rev. Lett. 80, 2245 (1998).

[23] A. J. Berkley, H. Xu, R. C. Ramos, M. A. Gubrud, F. W. Strauch, P. R. Johnson, J. R. Anderson, A. J. Dragt, C. J. Lobb, and F. C. Wellstood, Science 300, 1548 (2003).

[24] T. Yamamoto, Y. A. Pashkin, O. Astafiev, Y. Nakamura, and J. S. Tsai, Nature 425, 941 (2003).

[25] X. Li, Y. Wu, D. Steel, D. Gammon, T. H. Stievater, D. S.
Katzer, D. Park, C. Piermarocchi, and L. J. Sham, Science 301, 809 (2003).

[26] I. A. Grigorenko and D. V. Khveshchenko, Phys. Rev. Lett. 94, 040506 (2005).

[27] Y. M. Galperin, D. V. Shantsev, J. Bergli, and B. L. Altshuler, Europhys. Lett. 71, 21 (2005).

[28] R. H. Dicke, Phys. Rev. 93, 99 (1954).

[29] M. I. Shirokov, J. phys. B.: At. Mol. Phys. 23, 1923 (1990).

[30] A. Messiah, Quantum Mechanics (Dover, 1999).

[31] G. Morigi, S. Franke-Arnold, and G.-L. Oppo, Phys. Rev. A 66, 053409 (2002).

[32] E. del Valle, S. Zippilli, F. P. Laussy, A. Gonzalez-Tudela, G. Morigi, and C. Tejedor, Phys. Rev. B 81, 035302 (2010).

[33] B. D. Gerardot, S. Strauf, M. J. A. de Dood, A. M. Bychkov, A. Badolato, K. Hennessy, E. L. Hu, D. Bouwmeester, and P. M. Petroff, Phys. Rev. Lett. 95, 137403 (2005).

[34] H. J. Carmichael, Statistical methods in quantum optics 1 (Springer, 2002), 2nd ed.

[35] H.-J. Briegel and B.-G. Englert, Phys. Rev. A 47, 3311 (1993).

[36] E. del Valle, F. P. Laussy, and C. Tejedor, AIP Conference Proceedings 1147, 238 (2009).

[37] F. P. Laussy, E. del Valle, and C. Tejedor, Phys. Rev. Lett. 101, 083601 (2008).

[38] A. Laucht, N. Hauke, J. M. Villas-Bôas, F. Hofbauer, G. Böhm, M. Kaniber, and J. J. Finley, Phys. Rev. Lett. 103, 087405 (2009).

[39] When taking square roots, we always follow the prescription $\sqrt{c^{2}}=c$, for $c \in \mathbb{C}$. Considering this solution is enough because in any case all quantities depend on both $\pm \sqrt{c^{2}}$.

[40] That is, pictorially, when the structure of levels and transitions are rotated by $180^{\circ}$ or, mathematically, when the rising and lowering operators are inverted. 